Portland State University

PDXScholar

Mathematics and Statistics Faculty

Fariborz Maseeh Department of Mathematics

Publications and Presentations

and Statistics

2000

\title{
Multigrid for the Mortar Finite Element Method
}

Jay Gopalakrishnan

Portland State University, gjay@pdx.edu

Joseph E. Pasciak

Texas A \& M University - College Station

Follow this and additional works at: https://pdxscholar.library.pdx.edu/mth_fac

Part of the Analysis Commons, and the Applied Mathematics Commons

Let us know how access to this document benefits you.

\section{Citation Details}

Gopalakrishnan, J., \& Pasciak, J. E. (2000). Multigrid for the Mortar Finite Element Method. SIAM Journal On Numerical Analysis, 37(3), 1029.

This Article is brought to you for free and open access. It has been accepted for inclusion in Mathematics and Statistics Faculty Publications and Presentations by an authorized administrator of PDXScholar. Please contact us if we can make this document more accessible: pdxscholar@pdx.edu. 


\title{
MULTIGRID FOR THE MORTAR FINITE ELEMENT METHOD*
}

\author{
JAYADEEP GOPALAKRISHNAN ${ }^{\dagger}$ AND JOSEPH E. PASCIAK ${ }^{\dagger}$
}

\begin{abstract}
A multigrid technique for uniformly preconditioning linear systems arising from a mortar finite element discretization of second order elliptic boundary value problems is described and analyzed. These problems are posed on domains partitioned into subdomains, each of which is independently triangulated in a multilevel fashion. The multilevel mortar finite element spaces based on such triangulations (which need not align across subdomain interfaces) are in general not nested. Suitable grid transfer operators and smoothers are developed which lead to a variable Vcycle preconditioner resulting in a uniformly preconditioned algebraic system. Computational results illustrating the theory are also presented.
\end{abstract}

Key words. mortar, finite element method, multigrid, V-cycle, preconditioning, domain decomposition

AMS subject classifications. $65 \mathrm{~F} 10,65 \mathrm{~N} 55,65 \mathrm{~N} 30$

PII. S0036142998341040

1. Introduction. The mortar finite element method is a nonconforming domain decomposition technique tailored to handle problems posed on domains that are partitioned into independently triangulated subdomains. The meshes on different subdomains need not align across subdomain interfaces. The flexibility this technique offers by allowing substructures of a complicated domain to be meshed independently of each other is well recognized. In this paper we consider preconditioned iteration for the solution of the resulting algebraic system. Our preconditioner is a nonvariational multigrid procedure.

The mortar finite element discretization is a discontinuous Galerkin approximation. The functions in the approximation subspaces have jumps across subdomain interfaces and are standard finite element functions when restricted to the subdomains. The jumps across subdomain interfaces are constrained by conditions associated with one of the two neighboring meshes. Bernardi, Maday, and Patera (see [3, 4]) proved the coercivity of the associated bilinear form on the mortar finite element space, thus implying existence and uniqueness of solutions to the discrete problem. They also showed that the mortar finite element method is as accurate as the usual finite element method. Recently, stability and convergence estimates for an $h p$ version of the mortar finite element method were proved [19].

When each subdomain has a multilevel mesh, preconditioners for the linear system arising from the mortar discretization can be developed by multilevel techniques. A hierarchical preconditioner with conditioning which grows like the square of the number of levels is described in [11]. Substructuring preconditioners have also been developed for the mortar method [1]. In this paper, we show that a variable V-cycle may be used to develop a preconditioned system whose condition number remains bounded independently of the number of levels. A multigrid preconditioner for the

*Received by the editors June 24, 1998; accepted for publication (in revised form) April 14, 1999; published electronically March 8, 2000. This work was supported by the National Science Foundation under grant DMS 9626567, the Environmental Protection Agency under grant R 825207, and the State of Texas under ARP/ATP grant 010366-168.

http://www.siam.org/journals/sinum/37-3/34104.html

${ }^{\dagger}$ Department of Mathematics, Texas A\&M University, College Station, TX 77843 (jay@isc.tamu. edu, pasciak@math.tamu.edu). 
mortar method viewed as a saddle point problem is described in [5]. In contrast, our algorithm gives rise to a positive definite preconditioned system.

One of the difficulties in constructing a multigrid preconditioner for the mortar finite element method arises due to the fact that the multilevel mortar finite element spaces are, in general, not nested. Multigrid theory for nonnested spaces [7] may be employed to construct a variable V-cycle preconditioner, provided a suitable prolongation operator can be designed. We construct such a prolongation operator and prove that it satisfies the "regularity and approximation" property (Condition (C.2)) required for application of the multigrid theory.

The next difficulty is in the design of a smoother. Our smoother is based on the point Jacobi method. Its analysis is nonstandard since the constraints at subdomain interface gives rise to mortar basis functions with nonlocal support. We prove that these basis functions decay exponentially away from their nodal vertex. This leads to a strengthened Cauchy-Schwarz inequality which is used to verify the smoothing hypothesis (Condition (C.1)).

The remainder of the paper is organized as follows. Section 2 introduces most of the notation in the paper. Section 3 describes the multilevel mortar finite element spaces. In section 4 the variable $\mathrm{V}$-cycle multigrid algorithm is given and the main result (Theorem 4.1) is stated and proved. Section 5 provides proofs of some technical lemmas. Implementation issues are considered in section 6 while the results of numerical experiments illustrating the theory are given in section 7 .

2. Preliminaries. In this section, we provide some preliminaries and notation which will be used in the remainder of the paper. In addition, we describe the continuous problem and impose an assumption on the regularity of its solution.

Let $\Omega$ be an open subset of the plane. For nonnegative integers $s$, the Sobolev space $H^{s}(\Omega)$ (see $[10,14]$ ) is the set of functions in $L^{2}(\Omega)$ with distributional derivatives up to order $s$ also in $L^{2}(\Omega)$. If $s$ is a positive real number between nonnegative integers $m$ and $m+1, H^{s}(\Omega)$ is the space obtained by interpolation (by the real method [16]) between $H^{m}(\Omega)$ and $H^{m+1}(\Omega)$. The Sobolev norm on $H^{s}(\Omega)$ is denoted by $\|\cdot\|_{s, \Omega}$ and the corresponding Sobolev seminorm is denoted by $|\cdot|_{s, \Omega}$. For $\phi \in H^{s}(\Omega)$ and a segment $\gamma$ contained in $\bar{\Omega}$, the trace of $\phi$ on $\gamma$ is denoted by $\left.\phi\right|_{\gamma}$. We will often write $\|\phi\|_{r, \gamma}$ and $|\phi|_{r, \gamma}$ for the $H^{r}(\gamma)$ norm and seminorm, respectively, of the trace $\left.\phi\right|_{\gamma}$.

Assume that $\Omega$ is connected and that its boundary, $\partial \Omega$, is polygonal. Let $\partial \Omega$ be split into $\partial \Omega_{D}$ and $\partial \Omega_{N}$ such that $\partial \Omega=\bar{\partial}_{N} \cup \overline{\partial \Omega}_{D}$ and $\partial \Omega_{N} \cap \partial \Omega_{D}$ is empty and assume that $\partial \Omega_{D}$ has nonzero measure. Denote by $\mathcal{V}$ the subspace of the Sobolev space $H^{1}(\Omega)$ consisting of functions in $H^{1}(\Omega)$ whose trace on $\partial \Omega_{D}$ is zero. Denote by $\mathcal{V}^{\prime}$ the dual of the normed linear space $\mathcal{V}$. The dual norm $\|\cdot\|_{-1, \Omega}$ is defined by

$$
\|u\|_{-1, \Omega}=\sup _{\phi \in \mathcal{V}} \frac{\langle u, \phi\rangle}{\|\phi\|_{1, \Omega}},
$$

where $\langle\cdot, \cdot\rangle$ denotes the duality pairing. Note that $L^{2}(\Omega)$ is contained in $\mathcal{V}^{\prime}$ if we identify the functional $\langle v, \phi\rangle=(v, \phi)$ for all $v \in L^{2}(\Omega)$. Here $(\cdot, \cdot)$ denotes the innerproduct in $L^{2}(\Omega)$. For $-1<s<0,\|\cdot\|_{s, \Omega}$ is the norm on the space defined by interpolation between $\mathcal{V}^{\prime}$ and $L^{2}(\Omega)$.

We seek an approximate solution to the problem

$$
A(U, \phi)=F(\phi) \text { for all } \phi \in \mathcal{V},
$$


where $A(\cdot, \cdot)$ is bilinear form on $\mathcal{V} \times \mathcal{V}$ defined by

$$
A(u, v)=\int_{\Omega} \nabla u \cdot \nabla v d x
$$

and $F$ is a given continuous linear functional on $H^{1}(\Omega)$. This problem has a unique solution. For the mortar finite element method, we restrict our attention to $F$ of the form

$$
F(v)=\int_{\Omega} f v d x
$$

for $f \in L^{2}(\Omega)$. This is the variational form of the boundary value problem

$$
\begin{aligned}
-\Delta U & =f \text { in } \Omega, \\
U & =0 \text { on } \partial \Omega_{D}, \\
\frac{\partial U}{\partial n} & =0 \text { on } \partial \Omega_{N} .
\end{aligned}
$$

Although our results are stated for this model problem, extension to more general second order elliptic partial differential equations with more general boundary conditions are straightforward.

We will need to assume some regularity for solutions of Problem (2.1). We formalize it here into Assumption (A.1).

(A.1) There exists a $\beta$ in the interval $(1 / 2,1]$ for which

$$
\|U\|_{1+\beta, \Omega} \leq C\|F\|_{-1+\beta, \Omega}
$$

holds for solutions $U$ to the problem (2.1).

This is known to hold for a wide class of domains [15]. Note that we do not require full elliptic regularity ( $\beta=1$ case).

3. The mortar finite element method. In this section, we first provide notation for subdomains and triangulations. Next multilevel mortar finite element spaces are introduced and the mortar finite element problem is defined.

Partition $\Omega$ into nonoverlapping polygonal subdomains $\Omega_{i}, i=1, \ldots, K$. The interface $\Gamma=\cup_{i=1}^{K} \partial \Omega_{i} \backslash \partial \Omega$ is broken into a set of disjoint open straight line segments $\gamma_{k}$, each of which is contained in $\partial \Omega_{i} \cap \partial \Omega_{j}$ for some $i$ and $j$. The collection of these edges will be denoted by $Z$, i.e., $Z=\left\{\gamma_{1}, \gamma_{2}, \ldots, \gamma_{L}\right\}$.

Each $\Omega_{i}$ is triangulated to produce a quasi-uniform mesh $\mathcal{T}_{1}^{i}$ of size $h_{1}$. The triangulations generally do not align at the subdomain interfaces. We assume that the endpoints of each interface segment in $Z$ are vertices of $\mathcal{T}_{1}^{p}$ and $\mathcal{T}_{1}^{q}$ where $p$ and $q$ are such that $\gamma \subset \partial \Omega_{p} \cap \partial \Omega_{q}$. Denote the global mesh $\cup_{i} \mathcal{T}_{1}^{i}$ by $\mathcal{T}_{1}$. To set up the multigrid algorithm, we need a sequence of refinements of $\mathcal{T}_{1}$. We refine the triangulation $\mathcal{T}_{1}$ to produce $\mathcal{T}_{2}$ by splitting each triangle of $\mathcal{T}_{1}$ into four triangles by joining the midpoints of the edges of the triangle. The triangulation $\mathcal{T}_{2}$ is then quasiuniform of size $h_{2}=h_{1} / 2$. Repeating this process, we get a sequence of triangulations $\mathcal{T}_{k}, k=1, \ldots, J$, each quasi-uniform of size $h_{k}=h_{1} / 2^{k-1}$.

We next define the mortar finite element spaces following $[2,3,4,19]$ (our notation is close to that in [19]). First, we define spaces $\widetilde{\mathcal{V}}$ and $\widetilde{M}_{k}$ by

$$
\widetilde{\mathcal{V}}=\left\{v:\left.v\right|_{\Omega_{i}} \in H^{1}\left(\Omega_{i}\right) \quad \text { for all } i=1, \ldots, K, v=0 \text { on } \partial \Omega_{D}\right\}
$$


and

$$
\widetilde{M}_{k}=\left\{v \in \widetilde{\mathcal{V}}: v \text { is linear on each triangle of } \mathcal{T}_{k}\right\} .
$$

Throughout this paper we will use piecewise linear finite element spaces for convenience of notation. The results extend to higher order finite elements without difficulty [13].

For every straight line segment $\gamma \in Z$, there is an $i$ and $j$ such that $\gamma \subseteq \partial \Omega_{i} \cap \partial \Omega_{j}$. Assign one of $i$ and $j$ to be the mortar index, $M(\gamma)$, and the other then is the nonmortar index, $N M(\gamma)$. Let $\Omega_{M(\gamma)}$ denote the mortar domain of $\gamma$ and $\Omega_{N M(\gamma)}$ be the nonmortar domain of $\gamma$. For every $u \in \widetilde{\mathcal{V}}$ define $u_{\gamma}^{M}$ and $u_{\gamma}^{N M}$ to be the trace of $\left.u\right|_{\Omega_{M(\gamma)}}$ on $\gamma$ and the trace of $\left.u\right|_{\Omega_{N M(\gamma)}}$ on $\gamma$, respectively.

We now define two discrete spaces $S_{k}(\gamma)$ and $W_{k}(\gamma)$ on an interface segment $\gamma$. Every $\gamma \in Z$ can be divided into subintervals in two ways: by the vertices of the mesh in the mortar domain of $\gamma$ and by those of the nonmortar domain of $\gamma$. Consider $\bar{\gamma}$ as partitioned into subintervals by the vertices of the triangulation on nonmortar side. Let these vertices be denoted by $x_{k, \gamma}^{i}, i=0, \ldots, N$. Denote the subintervals $\left[x_{k, \gamma}^{i-1}, x_{k, \gamma}^{i}\right]$ by $\omega_{k, i}, i=1, \ldots, N$, where $\omega_{k, 1}$ and $\omega_{k, N}$ are the subintervals that are at the ends of $\gamma$. The discrete space $S_{k}(\gamma)$ is defined as follows:

$$
S_{k}(\gamma)=\left\{\begin{array}{l}
v \text { is linear on each } \omega_{k, i}, i=1, \ldots, N, \\
v: \quad v \text { is constant on } \omega_{k, 1} \text { and on } \omega_{k, N}, \\
\text { and } v \text { is continuous on } \gamma .
\end{array}\right\} .
$$

We also define the space $W_{k}(\gamma)$ by

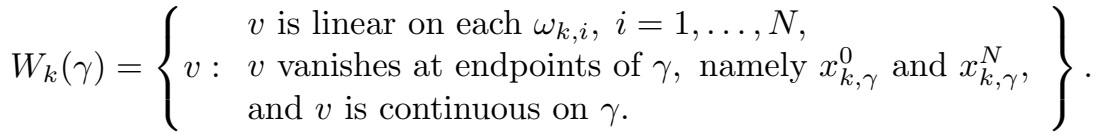

The multilevel mortar finite element spaces $M_{k}, k=1, \ldots, J$, are now defined by

$$
M_{k}=\left\{u \in \widetilde{M}_{k}: \begin{array}{l}
\text { on each } \gamma \in Z, \int_{\gamma}\left(u_{\gamma}^{M}-u_{\gamma}^{N M}\right) \chi d s=0 \\
\text { for all } \chi \in S_{k}(\gamma) .
\end{array}\right\} .
$$

The "mortaring" is done by constraining the jump across interfaces by the integral equality above. We will call this constraint the weak continuity of functions in $M_{k}$. Note that though the spaces $\left\{\widetilde{M}_{k}\right\}$ are nested,

$$
\widetilde{M}_{1} \subseteq \cdots \subseteq \widetilde{M}_{k} \subseteq \widetilde{M}_{k+1} \subseteq \cdots \subseteq \widetilde{M}_{J}
$$

the multilevel spaces $\left\{M_{k}\right\}$ are generally nonnested.

We next state the error estimates for the mortar finite element method. The mortar finite element approximation of the solution $U$ of Problem (2.1) (with $F$ given by (2.2)) is the function $U_{k} \in M_{k}$ satisfying

$$
\widetilde{A}\left(U_{k}, \phi\right)=\int_{\Omega} f \phi d x \quad \text { for all } \phi \in M_{k},
$$

where $\widetilde{A}(u, v)$ is the bilinear form on $\widetilde{\mathcal{V}} \times \widetilde{\mathcal{V}}$ defined by

$$
\widetilde{A}(u, v)=\sum_{i=1}^{K} \int_{\Omega_{i}} \nabla u \cdot \nabla v d x .
$$


It is shown in [3] that

$$
\|u\| \leq C \widetilde{A}(u, u) \quad \text { for all } u \in \widetilde{M}_{k},
$$

where $\|v\|^{2}=\sum_{i=1}^{K}\|v\|_{1, \Omega_{i}}^{2}$. Here and in the remainder of this paper, we will use $C$ to denote a generic constant independent of $h_{k}$ which can be different at different occurrences. It follows that (3.3) has a unique solution. It is also known (see [3]) that the mortar finite element approximation satisfies

$$
\left\|u-U_{k}\right\| \leq C h_{k}^{\beta}\|u\|_{1+\beta, \Omega} .
$$

We now define a projection, $\Pi_{k, \gamma}: L^{2}(\gamma) \rightarrow W_{k}(\gamma)$, which will be very useful in our analysis. For $u \in L^{2}(\gamma)$, it can be shown [4] that there exists a unique $v \in W_{k}(\gamma)$ satisfying

$$
\int_{\gamma} v \chi d s=\int_{\gamma} u \chi d s \quad \text { for all } \chi \in S_{k}(\gamma)
$$

We define $\Pi_{k, \gamma} u$ to be $v$. This projection is known to be stable in $L^{2}(\gamma)$ and $H_{0}^{1}(\gamma)$, i.e.,

$$
\begin{array}{ll}
\left\|\Pi_{k, \gamma} u\right\|_{0, \gamma} \leq C\|u\|_{0, \gamma} & \text { for all } u \in L^{2}(\gamma) \text { and } \\
\left\|\Pi_{k, \gamma} u\right\|_{1, \gamma} \leq C\|u\|_{1, \gamma} & \text { for all } u \in H_{0}^{1}(\gamma),
\end{array}
$$

under some weak assumptions on meshes (see [19]) which hold for the meshes defined above.

The projector $\Pi_{k, \gamma}$ is clearly related to the weak continuity condition. Let $\left\{y_{k}^{j}\right\}$ denote the nodes of $\mathcal{T}_{k}$ and the operator $\mathcal{E}_{k, \gamma}: \widetilde{\mathcal{V}} \rightarrow \widetilde{M}_{k}$ be defined by (see also Figure 3.1(a)-(d))

$$
\mathcal{E}_{k, \gamma} \widetilde{u}\left(y_{k}^{j}\right)= \begin{cases}\left(\Pi_{k, \gamma}\left(\widetilde{u}_{\gamma}^{M}-\widetilde{u}_{\gamma}^{N M}\right)\right)\left(y_{k}^{j}\right) & \text { if } y_{k}^{j} \in \gamma \cap \bar{\Omega}_{N M(\gamma)}, \\ 0 & \text { otherwise }\end{cases}
$$

It is easy to see that if $\widetilde{u}$ is in $\widetilde{M}_{k}$ then $u=\widetilde{u}+\sum_{\gamma \in Z} \mathcal{E}_{k, \gamma} \widetilde{u}$ is an element of $M_{k}$.

We next define a basis for $M_{k}$. Let $\left\{\widetilde{\phi}_{k}^{i}: i=1, \ldots, \widetilde{N}_{k}\right\}$ be the nodal basis for $\widetilde{M}_{k}$. There is more than one basis element associated with a node which appears in multiple subdomains. The basis for $M_{k}$ consists of functions of the form

$$
\phi_{k}^{i}=\widetilde{\phi}_{k}^{i}+\sum_{\gamma \in Z} \mathcal{E}_{k, \gamma}\left(\widetilde{\phi}_{k}^{i}\right)
$$

For every vertex $y_{k}^{l}$ located in the open segment $\gamma \in Z$ and belonging to the nonmortar side mesh, the corresponding $\phi_{k}^{l}$ as defined above is zero. Every remaining vertex $y_{k}^{l}$ leads to a nonzero $\phi_{k}^{l}$ since $\phi_{k}^{l}$ and $\widetilde{\phi}_{k}^{l}$ have the same nonzero value at $y_{k}^{l}$. Also, the values of $\phi_{k}^{l}$ and $\widetilde{\phi}_{k}^{l}$ at all nodes which are not nodes from nonmortar mesh lying in the interior of some $\gamma \in Z$ are the same. This implies that nonzero functions in $\left\{\phi_{k}^{i}\right\}$ are linearly independent. It is not difficult to check that these also form a basis for $M_{k}$. Since at $y_{k}^{l}, \phi_{k}^{l}$ is one and all other $\phi_{k}^{i}$ for $i \neq l$ are zero, these functions, in fact, form a nodal basis. Denote by $N_{k}$ the total number of nonzero $\phi_{k}^{i}$. We now reindex 


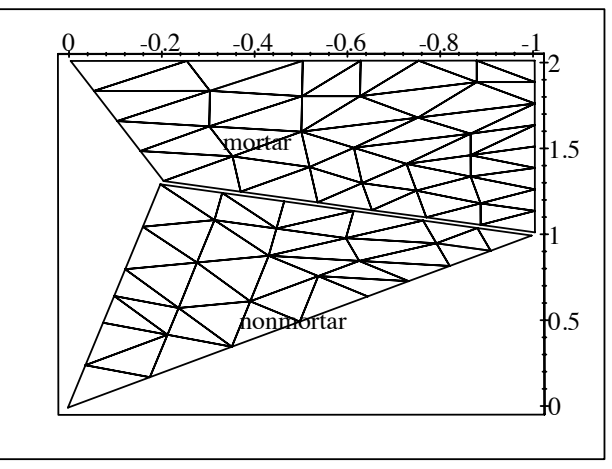

(a)

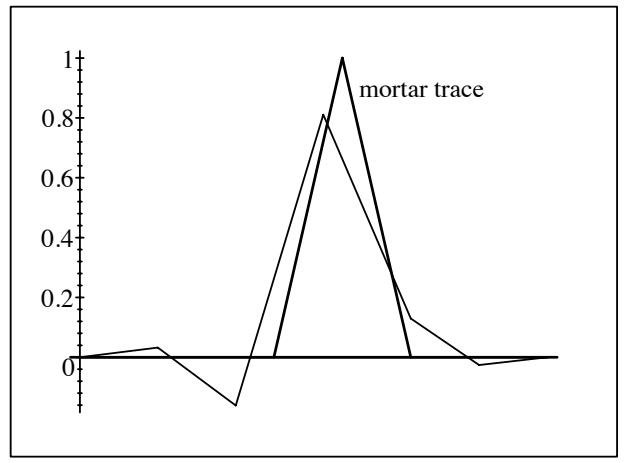

(c)

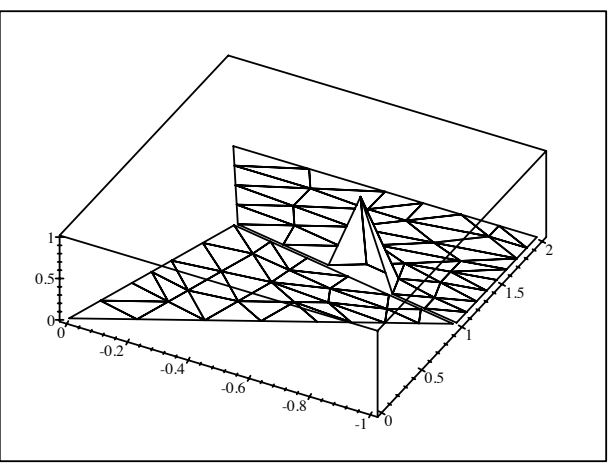

(b)

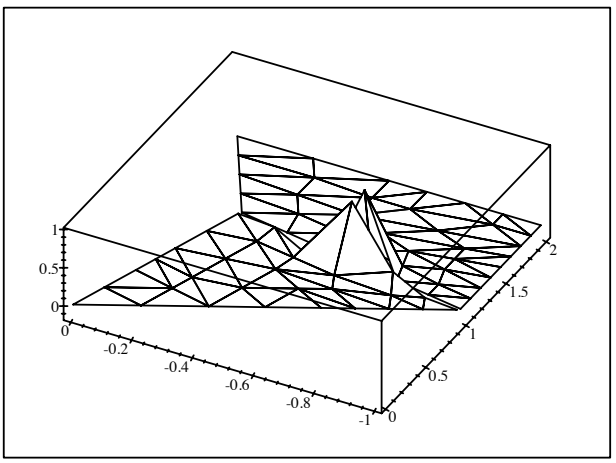

(d)

FIG. 3.1. Illustrating the action of $\mathcal{E}_{k, \gamma}$ : (a) Two subdomains with meshes that do not align at interface. (b) A discontinuous $\widetilde{u}$, which is 1 on a mortar node and 0 on the remaining nodes. (c) The thick line shows $\widetilde{u}_{\gamma}^{M}$ and the thin line shows $\Pi_{k, \gamma}\left(\widetilde{u}_{\gamma}^{M}-0\right)$. (d) Plot shows $\widetilde{u}+\mathcal{E}_{k, \gamma} \widetilde{u} . \mathcal{E}_{k, \gamma} \widetilde{u}$ is formed by extending $\Pi_{k, \gamma} \widetilde{u}_{\gamma}^{M}$ as described by (3.8).

$\left\{\widetilde{\phi}_{k}^{i}: i=1, \ldots, \widetilde{N}_{k}\right\}$ in such a way that every nonzero $\phi_{k}^{i}$ is in $\left\{\phi_{k}^{i}: i=1, \ldots, N_{k}\right\}$. We also reindex $\left\{y_{k}^{i}\right\}$ in this new ordering.

Now that we have a nodal basis for $M_{k}$, we may speak of the corresponding vertices of $\mathcal{T}_{k}$ as degrees of freedom for $M_{k}$. Consider an interface segment $\gamma \in Z$. All vertices on $\bar{\gamma}$ are degrees of freedom except (i) those on $\partial \Omega_{D}$ and (ii) those on $\gamma$ and from the nonmortar mesh. These are the vertices $y_{k}^{i}, i=1, \ldots, N_{k}$.

4. Multigrid algorithm for the mortar FEM. We will apply multigrid theory for nonnested spaces [7] to construct a variable V-cycle preconditioner. Before giving the algorithm, we define a prolongation operator and smoother. Later in this section, we will prove that our algorithm gives a preconditioner which results in a preconditioned system with uniformly bounded condition number.

First, let us establish some notation: $A_{k}$ will denote the operator on $M_{k}$, generated by the form $\widetilde{A}(\cdot, \cdot)$, i.e., $A_{k}$ is defined by

$$
\left(A_{k} u, v\right)=\widetilde{A}(u, v) \quad \text { for all } u, v \in M_{k} .
$$

The largest eigenvalue of $A_{k}$ is denoted by $\lambda_{k}$. For each basis element $\phi_{k}^{i}$, we define 
$M_{k}^{i}, i=1, \ldots, N_{k}$, to be the one dimensional subspace of $M_{k}$ spanned by $\phi_{k}^{i}$. Then

$$
M_{k}=\sum_{i=1}^{N_{k}} M_{k}^{i}
$$

provides a direct sum decomposition of $M_{k}$.

4.1. Smoothing and prolongation operators. We will use a smoother $R_{k}$ given by a scaled Jacobi method, i.e.,

$$
R_{k}=\alpha \sum_{i=1}^{N_{k}} A_{k, i}^{-1} Q_{k}^{i}
$$

where $\alpha$ is a positive constant to be chosen later. Here, $A_{k, i}: M_{k}^{i} \rightarrow M_{k}^{i}$ and $Q_{k}^{i}: L^{2}(\Omega) \rightarrow M_{k}^{i}$ are defined by

$$
\left(A_{k, i} v, \chi\right)=A(v, \chi) \quad \text { for all } \chi \in M_{k}^{i},
$$

and

$$
\left(Q_{k}^{i} v, \chi\right)=(v, \chi) \quad \text { for all } \chi \in M_{k}^{i},
$$

respectively. $R_{k}$ is symmetric in the $(\cdot, \cdot)$ inner-product. The following property of $R_{k}$ will be proved in section 5 .

(C.1) There exists a positive number $C_{R}$ independent of $k$ such that

$$
\frac{\|u\|_{0, \Omega}^{2}}{\lambda_{k}} \leq C_{R}\left(R_{k} u, u\right) \quad \text { for all } u \in M_{k} .
$$

In addition, $I-R_{k} A_{k}$ is nonnegative.

We now define "prolongation operators" $I_{k}: M_{k-1} \rightarrow M_{k}$, for $k=2, \ldots, J$. Clearly, $I_{k} u$ needs to satisfy the weak continuity constraint (see Definition 3.2). We define $I_{k} u$ by

$$
I_{k} u=u+\sum_{\gamma \in Z} \mathcal{E}_{k, \gamma}(u)
$$

In the next section we show that $I_{k}$ satisfies the following inequality.

(C.2) There exists a constant $C_{\beta}$ independent of $k$ such that

$$
\left|A_{k}\left(\left(I-I_{k} P_{k-1}\right) u, u\right)\right| \leq C_{\beta}^{2}\left(\frac{\left\|A_{k} u\right\|_{0, \Omega}^{2}}{\lambda_{k}}\right)^{\beta / 2}(\widetilde{A}(u, u))^{1-\beta / 2}
$$

for all $u$ in $M_{k}$.

Here $P_{k}$ is the $\widetilde{A}$-adjoint of $I_{k}$, i.e., $P_{k}: M_{k+1} \rightarrow M_{k}, k=1, \ldots, J-1$, satisfies

$$
\widetilde{A}\left(P_{k} u, \phi\right)=\widetilde{A}\left(u, I_{k+1} \phi\right) \quad \text { for all } \phi \in M_{k} .
$$

Condition (C.2) is verified using the regularity of the underlying partial differential equation. 
4.2. The algorithm. Let $m(k), k=1, \ldots, J$, be positive integers depending on $k$ and $P_{k-1}^{0}: M_{k} \rightarrow M_{k-1}$ be defined by

$$
\left(P_{k-1}^{0} u, v\right)=\left(u, I_{k} v\right) \quad \text { for all } u \in M_{k} \quad \text { and } \quad v \in M_{k-1} .
$$

The variable $\mathrm{V}$-cycle preconditioner $B_{k}$ for $k=1, \ldots, J$ is defined as follows.

Algorithm 4.2.

1. For $k=1$, set $B_{1}=A_{1}^{-1}$.

2. For $k=2 \ldots, J, B_{k} g$ for $g \in M_{k}$ is defined recursively by the following five steps.

(a) Set $x^{0}=0$.

(b) Define $x^{l}$, for $l=1, \ldots, m(k)$ by

$$
x^{l}=x^{l-1}+R_{k}\left(g-A_{k} x^{l-1}\right) .
$$

(c) Set $y^{0}=x^{m(k)}+I_{k} q$, where $q$ is given by

$$
q=B_{k-1} P_{k-1}^{0}\left(g-A_{k} x^{m(k)}\right) .
$$

(d) Define $y^{l}$ for $l=1, \ldots, m(k)$ by

$$
y^{l}=y^{l-1}+R_{k}\left(g-A_{k} y^{l-1}\right) .
$$

(e) Set $B_{k} g \equiv y^{m(k)}$.

We make the usual assumption on $m(k)$ (cf. [7]).

(A.2) The number of smoothings $m(k)$ increases as $k$ decreases in such a way that

$$
\beta_{0} m(k) \leq m(k-1) \leq \beta_{1} m(k)
$$

holds with $1<\beta_{0} \leq \beta_{1}$.

Typically $\beta_{1}$ is chosen so that the total work required for a multigrid cycle is no greater than the work required for application of the stiffness matrix on the finest level. This condition is satisfied, if for instance, $m(k)=2^{J-k}$.

The following theorem is the main result of this paper.

Theorem 4.1. Assume that (A.1) and (A.2) hold. There exists an $\alpha$ and $M>0$ independent of $J$ such that

$$
\eta^{-1} \widetilde{A}(u, u) \leq \widetilde{A}\left(B_{J} A_{J} u, u\right) \leq \eta \widetilde{A}(u, u) \quad \text { for all } u \in M_{J}
$$

with $\eta=\frac{M+m(J)^{\beta / 2}}{m(J)^{\beta / 2}}$.

The theorem shows that $B_{J}$ is a uniform preconditioner for the linear system arising from mortar finite element discretization using $M_{J}$ even if $m(J)=1$. Increasing $m(J)$ gives a somewhat better rate of convergence but increases the cost of applying $B_{J}$. It suffices to choose $\alpha$ above so that $\alpha<1 / C_{1}$ where $C_{1}$ is as in Lemma 4.5.

We use the following lemmas to prove Theorem 4.1. Their proofs will be given in section 5. First, we state a lemma that is a consequence of regularity which will be used in the proof of Condition (C.2).

Lemma 4.2. If (A.1) holds, then

$$
\left\|\left(I-I_{k} P_{k-1}\right) u\right\| \leq C h_{k}^{\beta}\left\|A_{k} u\right\|_{0, \Omega}^{\beta} \widetilde{A}(u, u)^{(1-\beta) / 2}
$$

holds for all $u$ in $M_{k}$. 
The next three lemmas are useful in analyzing the smoothing operator. We begin with a lemma from the theory of additive preconditioners.

Lemma 4.3. Let the space $V$ be a sum of subspaces $\sum_{i=1}^{l} V_{i}$. For $i=1,2, \ldots, l$, let $B_{i}$ be a symmetric positive definite operator on $V_{i}$ and $Q_{i}$ be the $L^{2}$ projection onto $V_{i}$. Then for $B=\sum_{i=1}^{l} B_{i} Q_{i}$,

$$
\left(B^{-1} u, u\right)=\inf _{\substack{u_{i} \in V_{i} \\ u=\sum_{i=1}^{l} u_{i}}}\left(\sum_{i=1}^{l}\left(B_{i}^{-1} u_{i}, u_{i}\right)\right)
$$

holds for all $u$ in $V$.

Lemma 4.3 may be found in [9] or stated in a different form in [17, Chapter 4]. We do not prove it here. The following two lemmas are used in the proof of Condition (C.1).

Lemma 4.4. For $R_{k}$ defined by (4.1), there exists a constant $C_{R}=C_{R}(\alpha)$ independent of $k$ such that (4.2) holds for all $u$ in $M_{k}$.

Lemma 4.5. For all $u$ in $M_{k}$, there is a number $C_{1}$ not depending on $J$ such that

$$
\left(A_{k} u, u\right) \leq C_{1} \sum_{i=1}^{N_{k}} c_{i}^{2} \widetilde{A}\left(\phi_{k}^{i}, \phi_{k}^{i}\right)
$$

where $u=\sum_{i=1}^{N_{k}} c_{i} \phi_{k}^{i}$ is the nodal basis decomposition.

We now prove the theorem.

Proof of Theorem 4.1. We apply the theorem for variable V-cycle in [6, Theorem 4.6]. This requires verification of Conditions (C.1) and (C.2).

Because of Lemma 4.4, (C.1) follows if we show that $I-R_{k} A_{k}$ is nonnegative, i.e., for all $u \in M_{k}$,

$$
\left(A_{k} R_{k} A_{k} u, u\right) \leq\left(A_{k} u, u\right) .
$$

This is equivalent to showing that for all $u \in M_{k}$,

$$
\left(A_{k} u, u\right) \leq\left(R_{k}^{-1} u, u\right) .
$$

Fix $u \in M_{k}$ and let $u=\sum_{i=1}^{N_{k}} c_{i} \phi_{k}^{i}$ be its nodal basis decomposition. Applying Lemma 4.3 gives

$$
\left(R_{k}^{-1} u, u\right)=\frac{1}{\alpha} \sum_{i=1}^{N_{k}}\left(A_{k, i} c_{i} \phi_{k}^{i}, c_{i} \phi_{k}^{i}\right)=\frac{1}{\alpha} \sum_{i=1}^{N_{k}} c_{i}^{2} \widetilde{A}\left(\phi_{k}^{i}, \phi_{k}^{i}\right) .
$$

The nonnegativity of $I-R_{k} A_{k}$ follows provided that $\alpha$ is taken to be less than or equal to $1 / C_{1}$ where $C_{1}$ is as in Lemma 4.5 .

Condition (C.2) is immediately seen to hold from Lemma 4.2. Indeed,

$$
\begin{aligned}
\widetilde{A}\left(\left(I-I_{k} P_{k-1}\right) u, u\right) & \leq C\left\|\left(I-I_{k} P_{k-1}\right) u\right\|\|u\| \\
& \leq C\left(\frac{\left\|A_{k} u\right\|_{0, \Omega}^{2}}{\lambda_{k}}\right)^{\beta / 2} \widetilde{A}(u, u)^{1-\beta / 2} .
\end{aligned}
$$

Here we have used the fact that $\lambda_{k} \leq C h_{k}^{-2}$. This proves (C.2) and thus completes the proof of the theorem. 
5. Proof of the lemmas. As a first step in proving Lemma 4.2, we prove that the operators $\left\{I_{k}\right\}$ approximate the identity operator $I$. After proving Lemma 4.2, we state and prove two lemmas used in the proof of Lemmas 4.4 and 4.5 .

Lemma 5.1. For any $u \in M_{k-1}$,

$$
\begin{aligned}
\left\|\left(I-I_{k}\right) u\right\| & \leq C \inf _{v \in \mathcal{V}}\|u-v\| \quad \text { and } \\
\left\|\left(I-I_{k}\right) u\right\|_{0, \Omega} & \leq C h_{k}\|u\| .
\end{aligned}
$$

Proof. Fix $u \in M_{k-1}$. By definition, $\left(I_{k}-I\right) u=\sum_{\gamma \in Z} \mathcal{E}_{k, \gamma} u$. Since $\mathcal{E}_{k, \gamma} u$ is zero on every interior vertex of the mesh in $\Omega_{N M(\gamma)}$,

$$
\left\|\mathcal{E}_{k, \gamma} u\right\|_{1, \Omega_{N M(\gamma)}}^{2} \approx \sum_{y_{k}^{i}}\left(\mathcal{E}_{k, \gamma} u\right)\left(y_{k}^{i}\right)^{2} \approx h_{k}^{-1}\left\|\mathcal{E}_{k, \gamma} u\right\|_{0, \gamma}^{2} .
$$

The above sum is taken over the vertices $y_{k}^{i}$ of the $\Omega_{N M(\gamma)}$ mesh that lie on $\gamma$. Here and elsewhere $\approx$ denotes equivalence with constants independent of $h_{k}$ and $\left\|\mathcal{E}_{k, \gamma} u\right\|_{0, \gamma}$ denotes the $L^{2}(\gamma)$ norm of the nonmortar trace of $\mathcal{E}_{k, \gamma} u$. By the $L^{2}$ stability of $\Pi_{k, \gamma}$,

$$
\begin{aligned}
\left\|\mathcal{E}_{k, \gamma} u\right\|_{0, \gamma}^{2} & =\left\|\Pi_{k, \gamma}\left(u_{\gamma}^{M}-u_{\gamma}^{N M}\right)\right\|_{0, \gamma}^{2} \\
& \leq C\left\|u_{\gamma}^{M}-u_{\gamma}^{N M}\right\|_{0, \gamma}^{2} .
\end{aligned}
$$

Since $u$ is in $M_{k-1}$, denoting $u_{\gamma}^{M}-u_{\gamma}^{N M}$ by $e$, we have that for any $\nu \in S_{k-1}(\gamma)$,

$$
(e, e)_{\gamma}=(e, e-\nu)_{\gamma} \leq\|e\|_{0, \gamma}\|e-\gamma\|_{0, \gamma},
$$

where $(\cdot, \cdot)_{\gamma}$ denotes the $L^{2}(\gamma)$ inner-product. It then follows that

$$
\left\|\mathcal{E}_{k, \gamma} u\right\|_{0, \gamma} \leq \inf _{\nu \in S_{k-1}(\gamma)}\|e-\nu\|_{0, \gamma} .
$$

If $q$ denotes the $L^{2}$ orthogonal projection into $S_{k-1}(\gamma)$, then by the approximation properties of $S_{k-1}(\gamma)$, we have $\|e-q(e)\|_{0, \gamma} \leq C h_{k}\|e\|_{1, \gamma}$. Trivially, we also have that $\|e-q(e)\|_{0, \gamma} \leq\|e\|_{0, \gamma}$. Thus the theory of interpolation of operators gives that

$$
\left\|\mathcal{E}_{k, \gamma} u\right\|_{0, \gamma} \leq C h_{k}^{1 / 2}\left\|u_{\gamma}^{M}-u_{\gamma}^{N M}\right\|_{1 / 2, \gamma} .
$$

Since $u_{\gamma}^{M}-u_{\gamma}^{N M}$ is equal to $(u-v)_{\gamma}^{M}-(u-v)_{\gamma}^{N M}$ whenever $v$ is in $\mathcal{V}$, the first inequality of the lemma readily follows when (5.5) is combined with (5.3), and a trace theorem is applied.

To prove the last inequality of the lemma, we start with

$$
\left\|\left(I-I_{k}\right) u\right\|_{0, \Omega}^{2} \leq C h_{k} \sum_{\gamma \in Z}\left\|\mathcal{E}_{k, \gamma} u\right\|_{0, \gamma}^{2} .
$$

Applying (5.5), we get

$$
\left\|\left(I-I_{k}\right) u\right\|_{0, \Omega}^{2} \leq C h_{k}^{2} \sum_{\gamma \in Z}\left\|u_{\gamma}^{M}-u_{\gamma}^{N M}\right\|_{1 / 2, \gamma}^{2},
$$

from which (5.2) follows after using the triangle inequality and a trace theorem. 
Proof of Lemma 4.2. The proof is broken into two parts. First, we prove that

$$
\left\|\left(I-I_{k} P_{k-1}\right) u\right\| \leq C\left(h_{k}^{\beta}\left\|A_{k} u\right\|_{-1+\beta, \Omega}+h_{k}\left\|A_{k} u\right\|_{0, \Omega}\right)
$$

holds for all $u$ in $M_{k}$. Next, we show that

$$
\left\|A_{k} u\right\|_{-1+\beta, \Omega} \leq C \widetilde{A}(u, u)^{(1-\beta) / 2}\left\|A_{k} u\right\|_{0, \Omega}^{\beta}
$$

holds for all $u$ in $M_{k}$. Clearly the lemma follows using (5.7) to bound the first term on the right-hand side of (5.6) and the fact that $\lambda_{k} \leq C h_{k}^{-2}$.

Fix $u$ in $M_{k}$ and set $g=A_{k} u$. Then $u$ solves

$$
\widetilde{A}(u, \phi)=(g, \phi) \quad \text { for all } \phi \in M_{k} .
$$

Let $w \in \mathcal{V}$ be the solution of

$$
A(w, \phi)=(g, \phi) \quad \text { for all } \phi \in \mathcal{V} .
$$

Now $u$ is the mortar finite element approximation to $w$ from $M_{k}$ and hence by (3.4),

$$
\|u-w\| \leq C h_{k}^{\beta}\|w\|_{1+\beta, \Omega} .
$$

By the triangle inequality,

$$
\left\|u-I_{k} P_{k-1} u\right\| \leq C h_{k}^{\beta}\|w\|_{1+\beta, \Omega}+\left\|w-I_{k} P_{k-1} u\right\| .
$$

To estimate the second term of (5.10), we start by writing $P_{k-1} u=v_{1}+v_{2}$ where $v_{1} \in M_{k-1}$ solves

$$
\widetilde{A}\left(v_{1}, \phi\right)=(g, \phi) \quad \text { for all } \phi \in M_{k-1} .
$$

The remainder $v_{2}$ satisfies

$$
\widetilde{A}\left(v_{2}, \phi\right)=\left(g,\left(I_{k}-I\right) \phi\right) \quad \text { for all } \phi \in M_{k-1} .
$$

Then

$$
\begin{aligned}
\left\|w-I_{k} P_{k-1} u\right\| & \leq\left\|w-v_{1}\right\|+\left\|I_{k} v_{2}\right\|+\left\|\left(I-I_{k}\right) v_{1}\right\| \\
& \leq C\left\|w-v_{1}\right\|+C\left\|v_{2}\right\| \\
& \leq C h_{k}^{\beta}\|w\|_{1+\beta, \Omega}+C\left\|v_{2}\right\|,
\end{aligned}
$$

where we have used Lemma 5.1 and (3.4). Using the coercivity of the form $\widetilde{A}(\cdot, \cdot)$, we find from (5.11) that

$$
\begin{aligned}
\left\|v_{2}\right\|^{2} & \leq C \widetilde{A}\left(v_{2}, v_{2}\right)=C\left(A_{k} u,\left(I_{k}-I\right) v_{2}\right) \\
& \leq\left\|A_{k} u\right\|_{0, \Omega}\left\|\left(I-I_{k}\right) v_{2}\right\|_{0, \Omega} .
\end{aligned}
$$

The last inequality of Lemma 5.1 now yields that $\left\|v_{2}\right\| \leq C h_{k}\left\|A_{k} u\right\|_{0, \Omega}$. Combining these estimates we get that

$$
\left\|w-I_{k} P_{k-1} u\right\| \leq C h_{k}^{\beta}\|w\|_{1+\beta, \Omega}+C h_{k}\left\|A_{k} u\right\|_{0, \Omega} .
$$

Using this in (5.10) and applying Assumption (A.1) proves (5.6). 
We next prove (5.7). Fix $u$ in $M_{k}$. Since $\|\cdot\|_{-1+\beta, \Omega}$ is the norm on the space in the interpolation scale between $\mathcal{V}^{\prime}$ and $L^{2}(\Omega)$,

$$
\left\|A_{k} u\right\|_{-1+\beta, \Omega} \leq\left\|A_{k} u\right\|_{-1, \Omega}^{1-\beta}\left\|A_{k} u\right\|_{0, \Omega}^{\beta} .
$$

Thus, it suffices to prove that

$$
\left\|A_{k} u\right\|_{-1, \Omega} \leq C \widetilde{A}(u, u)^{1 / 2} .
$$

Given $\psi$ in $\mathcal{V}$, we will construct $\psi_{k}=\psi_{k}(\psi) \in M_{k}$ satisfying

$$
\begin{aligned}
\left\|\psi_{k}\right\| & \leq C\|\psi\|_{1, \Omega} \quad \text { and } \\
\left\|\psi-\psi_{k}\right\|_{0, \Omega} & \leq C h_{k}\|\psi\|_{1, \Omega} .
\end{aligned}
$$

Assuming such a $\psi_{k}$ exists, we have

$$
\begin{aligned}
\left\|A_{k} u\right\|_{-1, \Omega} & =\sup _{\psi \in \mathcal{V}} \frac{\left(A_{k} u, \psi\right)}{\|\psi\|_{1, \Omega}} \\
& \leq \sup _{\psi \in \mathcal{V}} \frac{\left(A_{k} u, \psi-\psi_{k}\right)}{\|\psi\|_{1, \Omega}}+\sup _{\psi \in \mathcal{V}} \frac{\left(A_{k} u, \psi_{k}\right)}{\|\psi\|_{1, \Omega}} .
\end{aligned}
$$

Inequality (5.12) then follows from

$$
\begin{aligned}
\left\|A_{k} u\right\|_{-1, \Omega} & \leq \sup _{\psi \in \mathcal{V}} \frac{\left\|A_{k} u\right\|_{0, \Omega}\left\|\psi-\psi_{k}\right\|_{0, \Omega}}{\|\psi\|_{1, \Omega}}+\sup _{\psi \in \mathcal{V}} \frac{\widetilde{A}\left(u, \psi_{k}\right)}{\|\psi\|_{1, \Omega}} \\
& \leq C\left(h_{k}\left\|A_{k} u\right\|_{0, \Omega}+\widetilde{A}(u, u)^{1 / 2} \sup _{\psi \in \mathcal{V}} \frac{\left\|\psi_{k}\right\|_{1, \Omega}}{\|\psi\|_{1, \Omega}}\right) \\
& \leq C h_{k} \lambda_{k}^{1 / 2} \widetilde{A}(u, u)^{1 / 2}+C \widetilde{A}(u, u)^{1 / 2} \\
& \leq C \widetilde{A}(u, u)^{1 / 2} .
\end{aligned}
$$

To complete the proof, we need only construct $\psi_{k}$ satisfying (5.13) and (5.14). For $\psi \in \mathcal{V}$, let $\widetilde{\psi}_{k} \in \widetilde{M}_{k}$ be the $L^{2}$ projection of $\psi$ into $\widetilde{M}_{k}$. This projection is local on $\Omega_{i}$ and satisfies (see [8])

$$
\begin{aligned}
\left\|\widetilde{\psi}_{k}\right\| & \leq C|\psi|_{1, \Omega} \quad \text { and } \\
\left\|\psi-\widetilde{\psi}_{k}\right\|_{0, \Omega} & \leq C h_{k}|\psi|_{1, \Omega} .
\end{aligned}
$$

To construct $\psi_{k}$, we modify $\widetilde{\psi}_{k}$ so that the result is in $M_{k}$, i.e.,

$$
\psi_{k}=\widetilde{\psi}_{k}+\sum_{\gamma \in Z} \mathcal{E}_{k, \gamma}\left(\widetilde{\psi}_{k}\right)
$$

We will now show that $\psi_{k}$ defined above satisfies (5.13). We start with

$$
\left\|\psi_{k}\right\| \leq\left\|\widetilde{\psi}_{k}\right\| \mid+\sum_{\gamma \in Z}\left\|\mathcal{E}_{k, \gamma} \widetilde{\psi}_{k}\right\| .
$$


Using (5.15) on the first term on right-hand side and using (5.3) on the remaining, we get

$$
\left\|\psi_{k}\right\|^{2} \leq C\left(\|\psi\|_{1, \Omega}^{2}+h_{k}^{-1} \sum_{\gamma \in Z}\left\|\mathcal{E}_{k, \gamma} \widetilde{\psi}_{k}\right\|_{0, \gamma}^{2}\right)
$$

Note that $\left\|\mathcal{E}_{k, \gamma} \widetilde{\psi}_{k}\right\|_{0, \gamma} \leq C\left\|\left(\widetilde{\psi}_{k}\right)_{\gamma}^{M}-\left(\widetilde{\psi}_{k}\right)_{\gamma}^{N M}\right\|_{0, \gamma}$ by (5.4). Since $\psi$ is in $H^{1}(\Omega)$, its trace on $\gamma$ is in $L^{2}(\gamma)$. Moreover, $\psi_{\gamma}^{M}$ and $\psi_{\gamma}^{N M}$ are equal. Hence,

$$
\begin{aligned}
\left\|\mathcal{E}_{k, \gamma} \widetilde{\psi}_{k}\right\|_{0, \gamma} \leq & C\left\|\left(\widetilde{\psi}_{k}-\psi\right)_{\gamma}^{M}-\left(\widetilde{\psi}_{k}-\psi\right)_{\gamma}^{N M}\right\|_{0, \gamma} \\
\leq & C\left\|\widetilde{\psi}_{k}-\psi\right\|_{0, \Omega_{M(\gamma)}}^{1 / 2}\left\|\widetilde{\psi}_{k}-\psi\right\|_{1, \Omega_{M(\gamma)}}^{1 / 2} \\
& +C\left\|\widetilde{\psi}_{k}-\psi\right\|_{0, \Omega_{N M(\gamma)}}^{1 / 2}\left\|\widetilde{\psi}_{k}-\psi\right\|_{1, \Omega_{N M(\gamma)}}^{1 / 2},
\end{aligned}
$$

where in the last step we have used a trace inequality. Using (5.15) and (5.16), we then have

$$
\left\|\mathcal{E}_{k, \gamma} \widetilde{\psi}_{k}\right\|_{0, \gamma} \leq C h_{k}^{1 / 2}\left(\|\psi\|_{1, \Omega_{M(\gamma)}}+\|\psi\|_{1, \Omega_{N M(\gamma)}}\right) .
$$

Combining (5.18) and (5.17) gives (5.13).

It now remains only to prove (5.14). By the triangle inequality,

$$
\left\|\psi-\psi_{k}\right\|_{0, \Omega} \leq\left\|\psi-\widetilde{\psi}_{k}\right\|_{0, \Omega}+\left\|\widetilde{\psi}_{k}-\psi_{k}\right\|_{0, \Omega} .
$$

The first term on the right-hand side is readily bounded as required by (5.16). For the second term, as in (5.3),

$$
\left\|\widetilde{\psi}_{k}-\psi_{k}\right\|_{0, \Omega} \leq C h_{k}^{1 / 2} \sum_{\gamma \in Z}\left\|\mathcal{E}_{k, \gamma} \widetilde{\psi}_{k}\right\|_{0, \gamma} .
$$

Inequality (5.14) now follows immediately from (5.18). This completes the proof of Lemma 4.2. $\quad \square$

We are left to prove the lemmas involving the smoother $R_{k}$. A critical ingredient in this analysis involves the decay properties of the projector $\Pi_{k, \gamma}$ away from the support of the data. Specifically, we use the following lemma.

LEMma 5.2. Let the support of $v \in L^{2}(\gamma)$ be contained in a connected subset $\sigma \subseteq \gamma$. Then there is a constant $c$ such that for any set $\kappa \subseteq \gamma$ disjoint from $\sigma$,

$$
\left\|\Pi_{k, \gamma} v\right\|_{0, \kappa} \leq C \exp \left(-c \frac{\operatorname{dist}(\kappa, \sigma)}{h_{k}}\right)\|v\|_{0, \gamma},
$$

where $\operatorname{dist}(\kappa, \sigma)$ is the distance between the sets $\kappa$ and $\sigma$.

Remark 5.3. Estimates similar to those in the above lemma for the $L^{2}$-orthogonal projection were given by Descloux [12]. Note that $\Pi_{k, \gamma}$ is not an $L^{2}$-orthogonal projection. For completeness, we include a proof for our case which is a modification of one given in [21, Chapter 5].

Proof. Recall that a $\gamma \in Z$ is partitioned into subintervals $\omega_{k, i}$ by the vertices $x_{k, \gamma}^{i}, i=0, \ldots, N$ of the mesh on $\Omega_{N M(\gamma)}$. Define the set $r_{0}$ as the union of those 


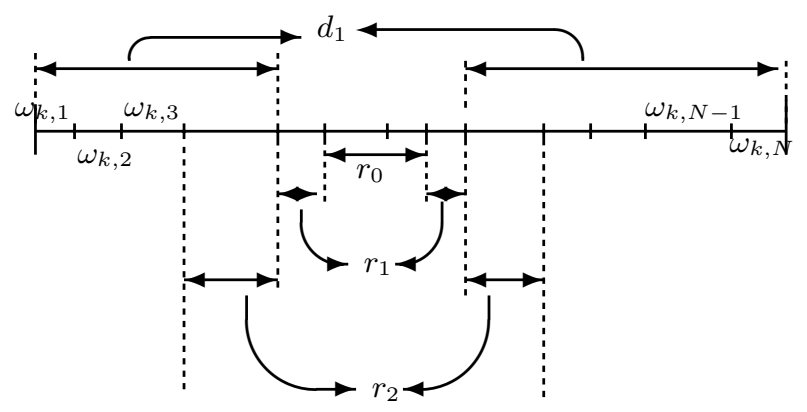

FIG. 5.1. An interface segment.

subintervals which intersect the support of $v$. Following the presentation in [21], define $r_{j}, j=1,2, \ldots$, recursively, by letting $r_{m}$ be the union of those subintervals of $\gamma$ that are not in $\cup_{l<m} r_{l}$ and which are neighbors of the subintervals of this set (see Figure 5.1). Further, let $d_{m}=\cup_{l>m} r_{l}$.

We will now show that the $L^{2}$ norm of $\Pi_{k, \gamma} v$ on $d_{m}$ can be bounded by a constant times its $L^{2}$ norm on $r_{m}$. For all $\chi \in S_{k}(\gamma)$ with support of $\chi$ disjoint from $r_{0}$, we have

$$
\left(\Pi_{k, \gamma} v, \chi\right)=(v, \chi)=0 .
$$

Let $\chi_{m} \in S_{k}(\gamma)$, for $m \geq 1$, be defined by

$$
\chi_{m}\left(x_{k, \gamma}^{j}\right)= \begin{cases}\Pi_{k, \gamma} v\left(x_{k, \gamma}^{j}\right) & \text { for } x_{k, \gamma}^{j} \in \bar{d}_{m}, \\ 0 & \text { otherwise }\end{cases}
$$

for $j=1, \ldots, N-1$. Let $\varepsilon=\omega_{k, 1} \cup \omega_{k, N}$. Clearly, (5.19) holds with $\chi_{m}$ in place of $\chi$. Moreover, $\chi_{m}(x)=\Pi_{k, \gamma} v(x)$ for $x \in d_{m} \backslash \varepsilon$, and it vanishes on $\gamma \backslash d_{m-1}$. Then

$$
0=\left(\chi_{m}, \Pi_{k, \gamma} v\right)=\int_{d_{m} \backslash \varepsilon}\left|\Pi_{k, \gamma} v\right|^{2} d s+\int_{d_{m} \cap \varepsilon} \chi_{m} \Pi_{k, \gamma} v d s+\int_{r_{m}} \chi_{m} \Pi_{k, \gamma} v d s .
$$

Note that on each subinterval of $d_{m} \cap \varepsilon, \chi_{m}$ is constant, and it takes the value of $\Pi_{k, \gamma} v$ at the interior endpoint. Also, on the subintervals of $r_{m}, \chi_{m}$ is either identically zero (if that subinterval is part of $r_{m} \cap \varepsilon$ ) or takes the value of $\Pi_{k, \gamma} v$ on one endpoint and zero on the other endpoint. From these observations, it is easy to conclude that

$$
\int_{d_{m} \cap \varepsilon} \chi_{m} \Pi_{k, \gamma} v d s \geq C\left\|\Pi_{k, \gamma} v\right\|_{0, \varepsilon \cap d_{m}}^{2}
$$

and

$$
\int_{r_{m}}\left|\Pi_{k, \gamma} v\left\|\chi_{m} \mid d s \leq C\right\| \Pi_{k, \gamma} v \|_{0, r_{m}}^{2} .\right.
$$

Thus,

$$
\begin{aligned}
C\left\|\Pi_{k, \gamma} v\right\|_{0, d_{m}}^{2} & \leq \int_{d_{m} \backslash \varepsilon}\left|\Pi_{k, \gamma} v\right|^{2} d s+\int_{d_{m} \cap \varepsilon} \chi_{m} \Pi_{k, \gamma} v d s \\
& =-\int_{r_{m}} \chi_{m} \Pi_{k, \gamma} v d s \leq C\left\|\Pi_{k, \gamma} v\right\|_{0, r_{m}}^{2} .
\end{aligned}
$$


Letting $q_{m}=\left\|\Pi_{k, \gamma} v\right\|_{0, d_{m}}^{2}$, the above inequality can be rewritten as $q_{m} \leq$ $C\left(q_{m-1}-q_{m}\right)$. It immediately follows that

$$
q_{m} \leq \frac{C}{1+C} q_{m-1} \leq \cdots \leq\left(\frac{C}{1+C}\right)^{m}\left\|\Pi_{k, \gamma} v\right\|_{0, \gamma}^{2} .
$$

The lemma easily follows from (3.6) and the observation that the distance between $\kappa$ and $\sigma$ is $O(m h)$.

Proof of Lemma 4.4. Fix $u \in M_{k}$ and let $u=\sum_{i=1}^{N_{k}} c_{i} \phi_{k}^{i}$ be the nodal basis decomposition. By Lemma 4.3,

$$
\begin{aligned}
\left(R_{k}^{-1} u, u\right) & =\frac{1}{\alpha} \sum_{i=1}^{l}\left(A_{k, i}\left(c_{i} \phi_{k}^{i}\right), c_{i} \phi_{k}^{i}\right) \\
& \leq \frac{\lambda_{k}}{\alpha} \sum_{i=1}^{l} c_{i}^{2}\left(\phi_{k}^{i}, \phi_{k}^{i}\right) .
\end{aligned}
$$

Note that the $L^{2}$ norm of every basis function $\phi_{k}^{i}$ is $O\left(h_{k}^{2}\right)$. Indeed, this is a standard estimate for those basis functions that coincide with a usual finite element nodal basis function on a subdomain. For the remaining basis functions, this follows from the exponential decay given by Lemma 5.2. Thus,

$$
\left(R_{k}^{-1} u, u\right) \leq \frac{C \lambda_{k} h_{k}^{2}}{\alpha} \sum_{i=1}^{N_{k}} c_{i}^{2}
$$

On each subdomain $\Omega_{j}$ we have that

$$
\|u\|_{0, \Omega_{j}}^{2} \approx h_{k}^{2}\left(\sum_{i=1}^{\widetilde{N}_{k}} u\left(y_{k}^{i}\right)^{2}\right) .
$$

Combining the above inequalities gives

$$
\left(R_{k}^{-1} u, u\right) \leq \frac{C \lambda_{k}}{\alpha}\|u\|_{0, \Omega}^{2} .
$$

The above inequality is equivalent to (4.2) and thus completes the proof of the lemma.

The proof of Lemma 4.5 requires a strengthened Cauchy-Schwarz inequality which we provide in the next lemma. First, we introduce some notation. Define the index sets $\tilde{\mathcal{N}}_{k}^{\gamma}$ and $\mathcal{N}_{k}^{\gamma}$ by

$$
\begin{aligned}
& \tilde{\mathcal{N}}_{k}^{\gamma}=\left\{i: y_{k}^{i} \in \gamma \cap \bar{\Omega}_{N M(\gamma)}\right\}, \\
& \mathcal{N}_{k}^{\gamma}=\left\{i: y_{k}^{i} \in \bar{\gamma} \text { and } i \notin \widetilde{\mathcal{N}}_{k}^{\gamma}\right\} .
\end{aligned}
$$

Also denote the set $\cup\left\{\mathcal{N}_{k}^{\gamma}: \gamma \in Z\right\}$ by $\mathcal{N}_{k}^{\Gamma}$.

LEMma 5.4. Let $\phi_{k}^{i}$ and $\phi_{k}^{j}$ be two basis functions of $M_{k}$ with $i, j \in \mathcal{N}_{k}^{\Gamma}$. Let $y_{k}^{i}$ and $y_{k}^{j}$ be the corresponding vertices. Then $\widetilde{A}\left(\phi_{k}^{i}, \phi_{k}^{j}\right)$ satisfies

$$
\widetilde{A}\left(\phi_{k}^{i}, \phi_{k}^{j}\right) \leq C \exp \left(-c \frac{\left|y_{k}^{i}-y_{k}^{j}\right|}{h_{k}}\right) \widetilde{A}\left(\phi_{k}^{i}, \phi_{k}^{i}\right)^{1 / 2} \widetilde{A}\left(\phi_{k}^{j}, \phi_{k}^{j}\right)^{1 / 2}
$$


where $C$ and $c$ are constants independent of $k$.

Proof. First, consider the case when $y_{k}^{i}$ and $y_{k}^{j}$ are on a same open interface segment $\gamma \in Z$. Let $\Delta_{M}$ denote the set of triangles that have at least one vertex on $\bar{\gamma}$ and are contained in $\Omega_{M(\gamma)}$. Similarly let $\Delta_{N M}$ denote the set of triangles that have at least one vertex on $\bar{\gamma}$ and are contained in $\Omega_{N M(\gamma)}$. Since $\phi_{k}^{i}$ and $\phi_{k}^{j}$ are simultaneously nonzero only on triangles in $\Delta_{M}$ or $\Delta_{N M}$, we have

$$
\widetilde{A}\left(\phi_{k}^{i}, \phi_{k}^{j}\right)=\sum_{\tau \in \Delta_{M}} A_{\tau}\left(\phi_{k}^{i}, \phi_{k}^{j}\right)+\sum_{\tau \in \Delta_{N M}} A_{\tau}\left(\phi_{k}^{i}, \phi_{k}^{j}\right)
$$

where $A_{\tau}(u, v)=\int_{\tau} \nabla u \cdot \nabla v d x$. The first sum obviously satisfies the required inequality, because this sum is zero whenever $y_{k}^{i}$ and $y_{k}^{j}$ are not vertices of the same triangle in $\Delta_{M}$.

Now consider a triangle $\tau \in \Delta_{N M}$. Recall that $\gamma$ was subdivided by the nonmortar mesh into subintervals $\omega_{k, i}, i=1, \ldots, N$. Let $\omega_{\tau}$ denote the union of two or more of these subintervals which have the vertices of $\tau$ as an endpoint (see Figure 5.2). Then because $\phi_{k}^{i}$ and $\phi_{k}^{j}$ are zero at the vertices of $\tau$ that are not in $\omega_{\tau}$,

$$
A_{\tau}\left(\phi_{k}^{i}, \phi_{k}^{j}\right) \leq\left|\phi_{k}^{i}\right|_{1, \tau}\left|\phi_{k}^{j}\right|_{1, \tau} \leq h_{k}^{-1}\left\|\left(\phi_{k}^{i}\right)_{\gamma}^{N M}\right\|_{0, \omega_{\tau}}\left\|\left(\phi_{k}^{j}\right)_{\gamma}^{N M}\right\|_{0, \omega_{\tau}} .
$$

Now, recall that $\phi_{k}^{i}$ and $\phi_{k}^{j}$ are obtained from $\widetilde{\phi}_{k}^{i}$ and $\widetilde{\phi}_{k}^{j}$, respectively, as described by (3.9). Denote by $s_{i}$ and $s_{j}$ the supports of the nonzero traces $\left.\widetilde{\phi}_{k}^{i}\right|_{\gamma}$ and $\left.\widetilde{\phi}_{k}^{j}\right|_{\gamma}$, respectively. Then by Lemma 5.2 ,

$$
\begin{aligned}
A_{\tau}\left(\phi_{k}^{i}, \phi_{k}^{j}\right) \leq & C h_{k}^{-1} \exp \left(-\frac{c}{h_{k}}\left[\operatorname{dist}\left(s_{i}, \omega_{\tau}\right)+\operatorname{dist}\left(s_{j}, \omega_{\tau}\right)\right]\right)\left\|\widetilde{\phi}_{k}^{i}\right\|_{0, \gamma}\left\|\widetilde{\phi}_{k}^{j}\right\|_{0, \gamma} \\
& \leq C \exp \left(-\frac{c}{h_{k}}\left[\operatorname{dist}\left(s_{i}, \omega_{\tau}\right)+\operatorname{dist}\left(s_{j}, \omega_{\tau}\right)\right]\right)\left\|\widetilde{\phi}_{k}^{i}\right\|_{1, \Omega_{M(\gamma)}}\left\|\widetilde{\phi}_{k}^{j}\right\|_{1, \Omega_{M(\gamma)}}
\end{aligned}
$$

Now, if $\left|\omega_{\tau}\right|$ denotes the length of $\omega_{\tau}$, it may easily be seen that

$$
\operatorname{dist}\left(s_{i}, \omega_{\tau}\right)+\operatorname{dist}\left(s_{j}, \omega_{\tau}\right)+\left|\omega_{\tau}\right| \geq \operatorname{dist}\left(s_{i}, s_{j}\right) .
$$

Further, by quasi uniformity,

$$
\operatorname{dist}\left(s_{i}, s_{j}\right) \geq\left|y_{k}^{i}-y_{k}^{j}\right|-C h_{k}
$$

Split the sum over $\tau \in \Delta_{N M}$ in (5.21) into a sum over triangles which have a vertex lying in between $y_{k}^{i}$ and $y_{k}^{j}$ on $\gamma$, and a sum over the remaining triangles in $\Delta_{N M}$. We denote the former set of triangles as $\Delta_{N M}^{i n}$ and the latter as $\Delta_{N M}^{\text {out }}$. Note that the number of triangles in $\Delta_{N M}^{i n}$ is bounded by $C\left|y_{k}^{i}-y_{k}^{j}\right| / h_{k}$. yield

We first consider triangles in $\Delta_{N M}^{i n}$. The observations of the previous paragraph 


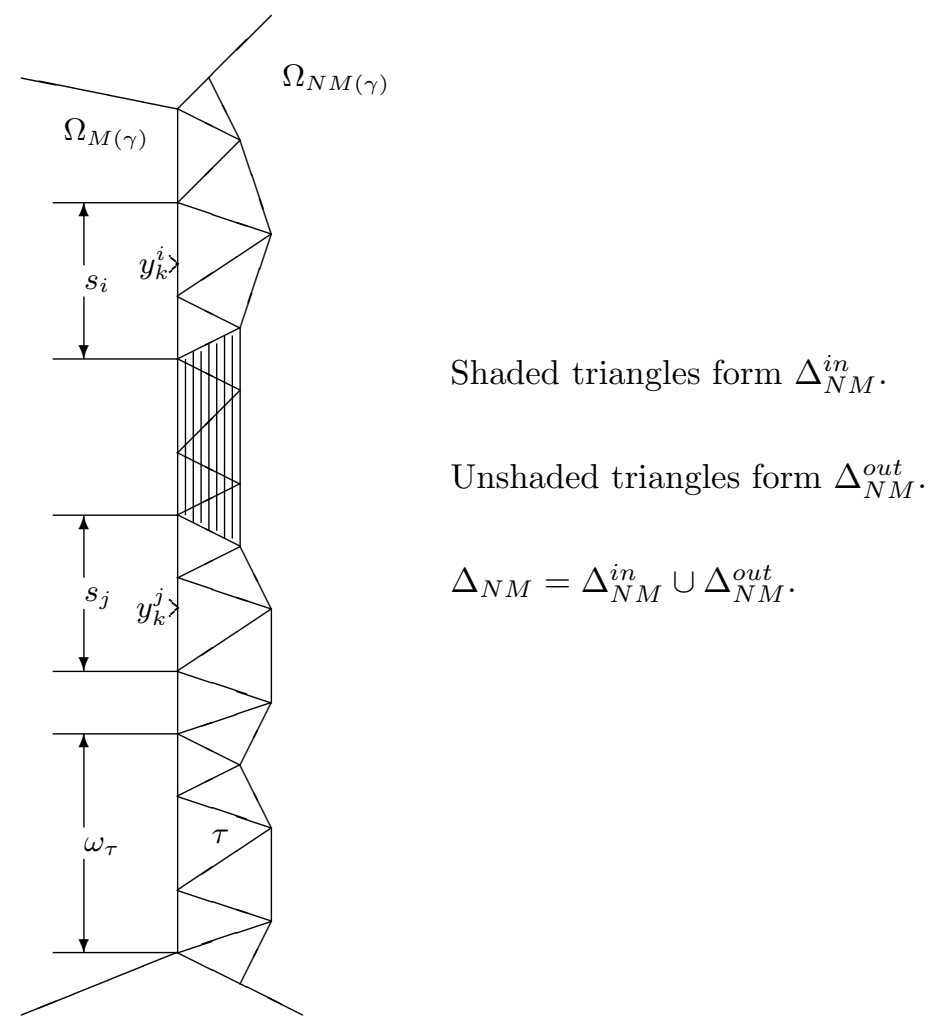

FIG. 5.2. Illustrating the notations in the proof of Lemma 5.4.

$$
\begin{aligned}
& \sum_{\tau \in \Delta_{N M}^{i n}} A_{\tau}\left(\phi_{k}^{i}, \phi_{k}^{j}\right) \\
& \quad \leq C \exp \left(-\frac{c}{h_{k}} \operatorname{dist}\left(s_{i}, s_{j}\right)\right)\left\|\widetilde{\phi}_{k}^{i}\right\|_{1, \Omega_{M(\gamma)}}\left\|\widetilde{\phi}_{k}^{j}\right\|_{1, \Omega_{M(\gamma)}}\left(\sum_{\tau \in \Delta_{N M}^{i n}} 1\right) \\
& \quad \leq C \frac{\left|y_{k}^{i}-y_{k}^{j}\right|}{h_{k}} \exp \left(-c \frac{\left|y_{k}^{i}-y_{k}^{j}\right|}{h_{k}}\right)\left\|\widetilde{\phi}_{k}^{i}\right\|_{1, \Omega_{M(\gamma)}}\left\|\widetilde{\phi}_{k}^{j}\right\|_{1, \Omega_{M(\gamma)}} \\
& \leq C \exp \left(-c \frac{\left|y_{k}^{i}-y_{k}^{j}\right|}{2 h_{k}}\right)\left\|\widetilde{\phi}_{k}^{i}\right\|_{1, \Omega_{M(\gamma)}}\left\|\widetilde{\phi}_{k}^{j}\right\|_{1, \Omega_{M(\gamma)}} .
\end{aligned}
$$

Now, for the sum over triangles in $\Delta_{N M}^{\text {out }}$, observe that one of the distances, $\operatorname{dist}\left(\omega_{\tau}, s_{i}\right)$ or $\operatorname{dist}\left(\omega_{\tau}, s_{j}\right)$, is greater than $\operatorname{dist}\left(s_{i}, s_{j}\right)$. Hence

$$
\begin{aligned}
\sum_{\tau \in \Delta_{N M}^{\text {out }}} A_{\tau}\left(\phi_{k}^{i}, \phi_{k}^{j}\right) \leq & C \exp \left(-c \frac{\left|y_{k}^{i}-y_{k}^{j}\right|}{h_{k}}\right)\left\|\widetilde{\phi}_{k}^{i}\right\|_{1, \Omega_{M(\gamma)}}\left\|\widetilde{\phi}_{k}^{j}\right\|_{1, \Omega_{M(\gamma)}} \\
& \times \sum_{\tau \in \Delta_{N M}^{\text {out }}} \exp \left(-c \frac{\operatorname{dist}\left(\omega_{\tau}, s_{i} \cup s_{j}\right)}{h_{k}}\right)
\end{aligned}
$$

Since the sum on the right-hand side can be bounded by a summable geometric series, 
we have

$$
\sum_{\tau \in \Delta_{N M}^{\text {out }}} A_{\tau}\left(\phi_{k}^{i}, \phi_{k}^{j}\right) \leq C \exp \left(-c \frac{\left|y_{k}^{i}-y_{k}^{j}\right|}{h_{k}}\right)\left\|\widetilde{\phi}_{k}^{i}\right\|_{1, \Omega_{M(\gamma)}}\left\|\widetilde{\phi}_{k}^{j}\right\|_{1, \Omega_{M(\gamma)}} .
$$

Thus, (5.23), (5.24), and (5.21) give

$$
\widetilde{A}\left(\phi_{k}^{i}, \phi_{k}^{j}\right) \leq C \exp \left(-c \frac{\left|y_{k}^{i}-y_{k}^{j}\right|}{2 h_{k}}\right)\left\|\phi_{k}^{i}\right\|\|\| \phi_{k}^{j} \| .
$$

This, along with the coercivity of $\widetilde{A}(\cdot, \cdot)$ on $M_{k} \times M_{k}$, proves the lemma when $y_{k}^{i}$ and $y_{k}^{j}$ lie on the same $\gamma$.

Now suppose one of $y_{k}^{i}$ and $y_{k}^{j}$, say $y_{k}^{i}$, is an endpoint of $\bar{\gamma}$. Then (5.22) holds for all $\tau \in \Delta_{N M}$, except possibly a triangle at all of whose vertices $\phi_{k}^{i}$ is nonzero. This triangle must have $y_{k}^{i}$ as one of its vertices. If $\tau$ is this triangle, then clearly

$$
A_{\tau}\left(\phi_{k}^{i}, \phi_{k}^{j}\right) \leq\left|\phi_{k}^{i}\right|_{1, \tau} h_{k}^{-1 / 2}\left|\phi_{k}^{j}\right|_{0, \omega_{\tau}} \leq C\left\|\phi_{k}^{i}\right\|_{1, \tau} \exp \left(-\frac{c}{h_{k}} \operatorname{dist}\left(s_{i}, s_{j}\right)\right)\left\|\widetilde{\phi}_{k}^{j}\right\|_{1, \Omega_{M(\gamma)}} .
$$

Hence, all the arguments for the previous case hold for this case too. The case when $y_{k}^{i}$ is one endpoint of $\gamma$ and $y_{k}^{j}$ is the other is handled in a similar fashion by considering separately the two possible triangles where (5.22) may not hold.

To conclude the proof, it now suffices to consider the case when $y_{k}^{i} \in \gamma_{1}$ and $y_{k}^{j} \in \gamma_{2}$ with $\gamma_{1} \neq \gamma_{2}$, and $\gamma_{1}, \gamma_{2} \in Z$. Then $\widetilde{A}\left(\phi_{k}^{i}, \phi_{k}^{j}\right)$ is zero unless there is a triangle $\tau$ in $\mathcal{T}_{k}$ which has one of its vertices contained in $\gamma_{1}$ and another contained in $\gamma_{2}$. It is easy to see that the required estimate follows in the latter case too.

Proof of Lemma 4.5. Split $u$ into a function $u_{0}$ that vanishes on the interface $\Gamma$ and a function $u_{\Gamma}$ that is a linear combination of $\phi_{k}^{i}$, with $i \in \mathcal{N}_{k}^{\Gamma}$. By the triangle inequality,

$$
\widetilde{A}(u, u) \leq 2\left[\widetilde{A}\left(u_{0}, u_{0}\right)+\widetilde{A}\left(u_{\Gamma}, u_{\Gamma}\right)\right] .
$$

On each triangle $\tau$ in $\mathcal{T}_{k}$,

$$
u_{0}=\sum_{j=1}^{3} c_{i(\tau ; j)} \phi_{k}^{i(\tau ; j)} \text { on } \tau,
$$

where $i(\tau ; j), j=1,2,3$, are the vertices of $\tau$. Applying the arithmetic-geometric mean inequality gives

$$
\begin{aligned}
\widetilde{A}\left(u_{0}, u_{0}\right) & =\sum_{\tau \in \mathcal{T}_{k}} A_{\tau}\left(u_{0}, u_{0}\right) \\
& \leq \sum_{\tau \in \mathcal{T}_{k}} 3 \sum_{j=1}^{3} c_{i(\tau ; j)}^{2} A_{\tau}\left(\phi_{k}^{i(\tau ; j)}, \phi_{k}^{i(\tau ; j)}\right) \\
& =3 \sum_{i \notin \mathcal{N}_{k}^{\Gamma}} c_{i}^{2} \sum_{\tau \in \mathcal{T}_{k}} A_{\tau}\left(\phi_{k}^{i}, \phi_{k}^{i}\right) \\
& =3 \sum_{i \notin \mathcal{N}_{k}^{\Gamma}} c_{i}^{2} \widetilde{A}\left(\phi_{k}^{i}, \phi_{k}^{i}\right) .
\end{aligned}
$$


All that remains is to estimate $\widetilde{A}\left(u_{\Gamma}, u_{\Gamma}\right)$. We clearly have

$$
\widetilde{A}\left(u_{\Gamma}, u_{\Gamma}\right)=\sum_{i, j \in \mathcal{N}_{\Gamma}} c_{i} c_{j} \widetilde{A}\left(\phi_{k}^{i}, \phi_{k}^{j}\right) .
$$

Applying Lemma 5.4 gives

$$
\begin{aligned}
\widetilde{A}\left(u_{\Gamma}, u_{\Gamma}\right) & \leq C \sum_{i, j \in \mathcal{N}_{k}^{\Gamma}} c_{i} c_{j} \exp \left(-c \frac{\left|y_{k}^{i}-y_{k}^{j}\right|}{h_{k}}\right) \widetilde{A}\left(\phi_{k}^{i}, \phi_{k}^{i}\right)^{1 / 2} \widetilde{A}\left(\phi_{k}^{j}, \phi_{k}^{j}\right)^{1 / 2} \\
& \leq C\|\mathbf{M}\|_{\ell^{2}} \sum_{i \in \mathcal{N}_{k}^{\Gamma}} c_{i}^{2} \widetilde{A}\left(\phi_{k}^{i}, \phi_{k}^{i}\right) .
\end{aligned}
$$

Here $\mathbf{M}$ is the matrix with entries

$$
\mathbf{M}_{i j}=\exp \left(-c \frac{\left|y_{k}^{i}-y_{k}^{j}\right|}{h_{k}}\right)
$$

and

$$
\|\mathbf{M}\|_{\ell^{2}}=\sup _{\zeta \in \mathbb{R}^{\left|\mathcal{N}_{k}^{\Gamma}\right|}} \frac{(\mathbf{M} \zeta) \cdot \zeta}{\zeta \cdot \zeta}
$$

where $\left|\mathcal{N}_{k}^{\Gamma}\right|$ denotes the cardinality of $\mathcal{N}_{k}^{\Gamma}$ and $\cdot$ indicates the standard dot product in $\mathbb{R}^{\left|\mathcal{N}_{k}^{\Gamma}\right|}$.

To conclude the proof, it suffices to show that $\|\mathbf{M}\|_{\ell^{2}}$ is bounded by a constant independent of $h_{k}$. Note that $\|\mathbf{M}\|_{\ell^{2}}$ is equal to the spectral radius of $\mathbf{M}$ and consequently, can be bounded by any induced norm. So,

$$
\|\mathbf{M}\|_{\ell^{2}} \leq \max _{i \in \mathcal{N}_{k}^{\Gamma}} \sum_{j \in \mathcal{N}_{k}^{\Gamma}} \mathbf{M}_{i j}
$$

For every fixed $i$, the sum on the right-hand side can be enlarged to run over all vertices of the mesh $\mathcal{T}_{k}$, and then one obtains

$$
\sum_{j \in \mathcal{N}_{k}^{\Gamma}} \mathbf{M}_{i j} \leq \sum_{y_{k}^{j} \in \mathcal{T}_{k}} \exp \left(-c \frac{\left|y_{k}^{j}-y_{k}^{i}\right|}{h_{k}}\right) \leq C \iint_{\mathbb{R}^{2}} \exp (-c|y|) d y .
$$

Thus, $\|\mathbf{M}\|_{\ell^{2}} \leq C$.

6. Implementation. This section will describe some details of implementing the mortar method and the preconditioner $B_{J}$. Since we shall be using a preconditioned iteration, all that is necessary is the implementation of the action of the stiffness matrix and that of the preconditioner.

Let $\mathbf{A}_{\widetilde{A}}$ denote the stiffness matrix for the mortar finite element method, i.e., $\left[\mathbf{A}_{k}\right]_{i j}=\widetilde{A}\left(\phi_{k}^{j}, \phi_{k}^{i}\right)$. Let

$$
v=\sum_{i=1}^{N_{k}} p_{i} \phi_{k}^{i}
$$

be an element of $M_{k}$. To apply $\mathbf{A}_{k}$ to $p=\left(p_{1}, \ldots, p_{N_{k}}\right)^{t}$ we first expand $v$ in the basis $\left\{\widetilde{\phi}_{k}^{i}\right\}$, apply the stiffness matrices for $\widetilde{M}_{k}$, and finally accumulate $\widetilde{A}\left(v, \phi_{k}^{i}\right), i=$ 
$1, \ldots, N_{k}$. The application of the stiffness matrix corresponding to the space $\widetilde{M}_{k}$ with nodal basis $\left\{\widetilde{\phi}_{k}^{i}\right\}$ is standard. As we shall see, the first and last steps are closely related.

The first step above involves computing the nodal representation of a function $v$ with respect to the basis $\left\{\widetilde{\phi}_{k}^{i}\right\}$ given the coefficients $\left\{p_{i}\right\}$ appearing in (6.1). Thus, we seek the vector $\widetilde{p}=\left(\widetilde{p}_{1}, \ldots, \widetilde{p}_{\widetilde{N}_{k}}\right)^{t}$ satisfying

$$
v=\sum_{j=1}^{\widetilde{N}_{k}} \widetilde{p}_{j} \widetilde{\phi}_{k}^{j} .
$$

Note that $\widetilde{p}_{j}=p_{j}$ for $j=1, \ldots, N_{k}$. Thus, we only need to determine the values of $\widetilde{p}_{j}$ for the remaining indices. These indices appear in some set $\widetilde{\mathcal{N}}_{k}^{\gamma}$ corresponding to one of the interface segments. We define the transfer matrix $\mathbf{T}^{k, \gamma}$ by

$$
\sum_{j \in \widetilde{\mathcal{N}}_{k}^{\gamma}} \mathbf{T}_{j i}^{k, \gamma} \widetilde{\phi}_{k}^{j}=\mathcal{E}_{k, \gamma} \widetilde{\phi}_{k}^{i} \quad \text { for all } i \in \mathcal{N}_{k}^{\gamma} .
$$

Then for $j \in \tilde{\mathcal{N}}_{k}^{\gamma}$,

$$
\widetilde{p}_{j}=\sum_{i \in \mathcal{N}_{k}^{\gamma}} \mathbf{T}_{j i}^{k, \gamma} p_{i}
$$

It therefore suffices to have the matrices $\mathbf{T}^{k, \gamma}$ in computations. Note that the entries of $\mathbf{T}^{k, \gamma}$ are obtained by inverting a mass matrix. This inversion can be done once and for all at the beginning of the computations, and the matrices $\mathbf{T}^{k, \gamma}$ can be stored. The storage space required is comparable to that of local stiffness matrices, as each $\mathbf{T}^{k, \gamma}$ is of size $\left|\mathcal{N}_{k}^{\gamma}\right| \times\left|\widetilde{\mathcal{N}}_{k}^{\gamma}\right|$.

The last step of accumulating $\widetilde{A}\left(v, \phi_{k}^{i}\right), i=1, \ldots, N_{k}$ is also implemented in terms of $\mathbf{T}^{k, \gamma}$. Given the results of the stiffness matrix evaluation on $\widetilde{M}_{k}$, i.e., the vector of values $\widetilde{A}\left(v, \widetilde{\phi}_{k}^{j}\right)$, we need to compute $\widetilde{A}\left(v, \phi_{k}^{i}\right)$. Clearly, $\widetilde{\phi}_{k}^{i}=\phi_{k}^{i}$ for nodes which are not on any of the interface segments, so we only need to compute $\widetilde{A}\left(v, \phi_{k}^{i}\right)$ for nodes such that $i \in \mathcal{N}_{k}^{\gamma}$ for some segment. This is given by

$$
\widetilde{A}\left(v, \phi_{k}^{i}\right)=\widetilde{A}\left(v, \widetilde{\phi}_{k}^{i}\right)+\sum_{\gamma} \sum_{j \in \widetilde{\mathcal{N}}_{k}^{\gamma}} \mathbf{T}_{j i}^{k, \gamma} \widetilde{A}\left(v, \widetilde{\phi}_{k}^{j}\right) .
$$

The sum on $\gamma$ above is over the segments with $i \in \mathcal{N}_{k}^{\gamma}$.

For convenient notation, let us denote by $\mathbf{T}_{k}$ the matrix of the linear process that takes $\left\{p_{i}: i=1, \ldots, N_{k}\right\}$ to $\left\{\widetilde{p}_{i}: i=1, \ldots, \widetilde{N}_{k}\right\}$. Then the matrix corresponding to $\left\{\widetilde{A}\left(v, \widetilde{\phi}_{k}^{i}\right)\right\} \rightarrow\left\{\widetilde{A}\left(v, \phi_{k}^{i}\right)\right\}$ is the transpose $\mathbf{T}_{k}^{t}$.

We now discuss the implementation of the preconditioner $B_{k}$. We use uppercase letters to denote vectors of inner-products and lowercase ones to denote vectors of coefficients. What is needed is a procedure that will compute the coefficients of $B_{k} g$ in the basis $\left\{\phi_{k}^{i}\right\}$, given the values $\left[G_{k}\right]_{i}=\left(g, \phi_{k}^{i}\right), i=1, \ldots, N_{k}$. The corresponding matrix will be denoted by $\mathbf{B}_{k}$. Clearly, $\mathbf{B}_{1}=\mathbf{A}_{1}^{-1}$. The matrix that takes a vector $\left\{\left(w, \phi_{k}^{i}\right)\right\}$ to coefficients of $R_{k} w$ with respect to $\left\{\phi_{k}^{i}\right\}$ will be denoted by $\mathbf{R}_{k}$. Finally, let $\mathbf{C}_{k}$ be the matrix associated with $I_{k}$, i.e., 


$$
I_{k} \phi_{k-1}^{i}=\sum_{j=1}^{N_{k}}\left[\mathbf{C}_{k}\right]_{i j} \phi_{k}^{j} .
$$

Assuming $\mathbf{B}_{k-1}$ has been defined, we define $\mathbf{B}_{k} G_{k}$ for $G_{k} \in \mathbb{R}^{N_{k}}$ by the following four steps:

1. Compute $x^{l}$ for $l=1, \ldots, m(k)$ by $x^{l}=x^{l-1}+\mathbf{R}_{k}\left(G_{k}-\mathbf{A}_{k} x^{l-1}\right)$.

2. Set $y^{0}=x^{m(k)}+\mathbf{C}_{k}^{t} q_{k-1}$, where $q_{k-1}=\mathbf{B}_{k-1} \mathbf{C}_{k}\left(G_{k}-\mathbf{A}_{k} x^{m(k)}\right)$.

3. Compute $y^{l}$ for $l=1, \ldots, m(k)$ by $y^{l}=y^{l-1}+\mathbf{R}_{k}\left(G_{k}-\mathbf{A}_{k} y^{l-1}\right)$.

4. Set $\mathbf{B}_{k} G_{k}=y^{m(k)}$.

This algorithm is straightforward to implement as a recursive procedure provided we have implementations of $\mathbf{R}_{k}, \mathbf{C}_{k}, \mathbf{C}_{k}^{t}, \mathbf{A}_{k}$, and $\mathbf{A}_{1}^{-1}$.

To compute $q_{k}=\mathbf{C}_{k}^{t} q_{k-1}$, we first let $\widetilde{q}_{k-1}=\mathbf{T}_{k-1} q_{k-1}$. We then apply the coarse to fine interpolation corresponding to the imbedding $\widetilde{M}_{k-1} \subset \widetilde{M}_{k}$. This gives a vector which we denote by $\widetilde{q}_{k}$. Then $q_{k}$ is given by the truncated vector $\left(\widetilde{q}_{k}^{1}, \ldots, \widetilde{q}_{k}^{N_{k}}\right)^{t}$.

To compute the action of the transpose, $G_{k-1}=\mathbf{C}_{k} G_{k}$, we start by defining $\widetilde{G}_{k}$ to be the vector which extends $G_{k}$ by $\widetilde{G}_{k}^{i}=0$ for $i>N_{k}$. Next we apply the adjoint of the coarse to fine imbedding $\left(\widetilde{M}_{k-1} \subset \widetilde{M}_{k}\right)$ to define the vector $\widetilde{G}_{k-1}$. Then $G_{k-1}=\mathbf{T}_{k-1}^{t} \widetilde{G}_{k-1}$.

Since our codes do not assemble matrices, we use the alternative smoother

$$
\underline{R}_{k} g=\Lambda_{k}^{-1} \sum_{i=1}^{N_{k}}\left(g, \phi_{k}^{i}\right) \phi_{k}^{i},
$$

where $\Lambda_{\widetilde{A}}$ is the largest eigenvalue of $\mathbf{A}_{k}$. This avoids the computation of the diagonal entry $\widetilde{A}\left(\phi_{k}^{i}, \phi_{k}^{i}\right)$. The corresponding matrix operator $\mathbf{R}_{k}$ is just multiplication by $\Lambda_{k}^{-1}$.

We now show that this operator is a good smoother by showing that it satisfies Condition (C.1). First, (4.2) holds for $\underline{R}_{k}$ since by Lemma 4.4,

$$
\begin{aligned}
\frac{\|v\|_{0, \Omega}^{2}}{\lambda_{k}} & \leq C_{R}\left(R_{k} v, v\right)=C_{R} \sum_{i=1}^{N_{k}} \frac{\left(v, \phi_{k}^{i}\right)^{2}}{\widetilde{A}\left(\phi_{k}^{i}, \phi_{k}^{i}\right)} \\
& \leq C \Lambda_{k}{ }^{-1} \sum_{i=1}^{N_{k}}\left(v, \phi_{k}^{i}\right)^{2}=C\left(\underline{R}_{k} v, v\right) .
\end{aligned}
$$

Now let $v$ be in $M_{k}$ and $p$ be as in (6.1). Then

$$
\begin{aligned}
\left(\underline{R}_{k} A_{k} v, A_{k} v\right) & =\Lambda_{k}^{-1} \sum_{i=1}^{N_{k}} \widetilde{A}\left(v, \phi_{k}^{i}\right)^{2} \\
& =\frac{\mathbf{A}_{k} p \cdot \mathbf{A}_{k} p}{\Lambda_{k}} \leq \mathbf{A}_{k} p \cdot p=\left(A_{k} v, v\right) .
\end{aligned}
$$

This shows that $I-\underline{R}_{k} A_{k}$ is nonnegative and hence Condition (C.1) is satisfied.

7. Numerical results. In this section we give the results of model computations which illustrate that the condition numbers of the preconditioned system remain bounded as the number of levels increase. The program used for computations, takes as input general triangulations generated independently on subdomains, recursively refines these triangulations by breaking each triangle into four similar ones, and solves a mortar finite element problem using the mortar multigrid preconditioner. 

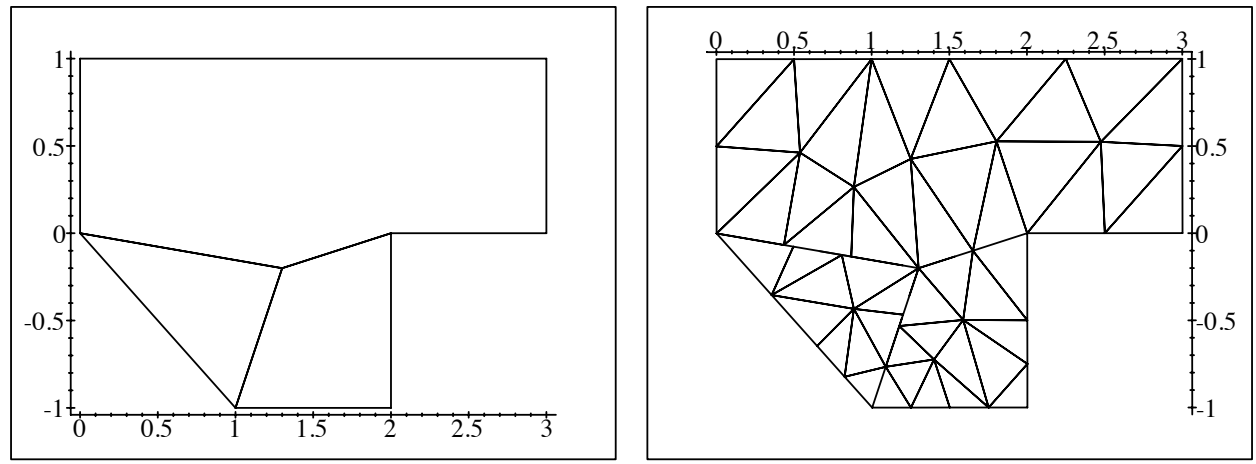

FIG. 7.1. Domain decomposition and initial triangulation for a domain with a re-entrant corner.
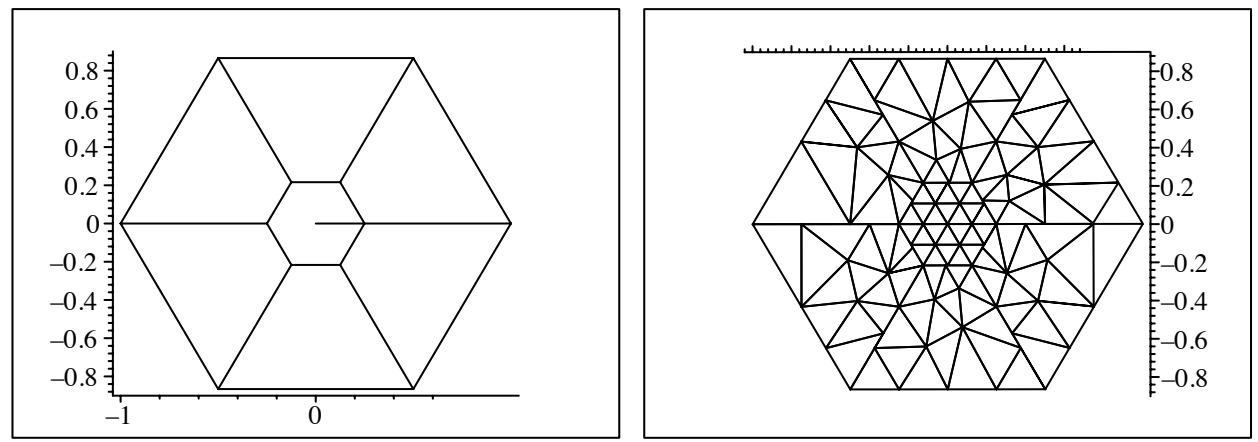

FIG. 7.2. Domain decomposition and initial triangulation for a domain with a cut.

We compute the mortar finite element approximation to a solution of the Poisson equation with zero Dirichlet boundary conditions on two domains. In both cases, the triangulations were done using the mesh generator TRIANGLE [20]. The smoother used was $\underline{R}_{k}$ defined in the previous section and $m(k)=2^{J-k}$. Estimates of extreme eigenvalues of the operator $\mathbf{B}_{J} \mathbf{A}_{J}$ were given by those of the Lanczos matrix (see [18]). Note that the eigenvalues of $\mathbf{B}_{J} \mathbf{A}_{J}$ coincide with those of $B_{J} A_{J}$.

Figure 7.1 shows the first domain, its decomposition into three subdomains, and the coarse meshes on subdomains. Note that this domain has a re-entrant corner. By virtue of a theorem in [15], solutions to Poisson equation on this domain satisfy Assumption (A.1) with $\beta<2 / 3$.

The domain decomposition and coarse triangulations of the second domain are pictured in Figure 7.2. This hexagonal domain has a cut that extends from the origin to a vertex of the hexagon. The domain is divided into seven subdomains. Assumption (A.1) does not hold for this domain.

Table 7.1 gives the condition numbers of $\mathbf{B}_{J} \mathbf{A}_{J}$ for the first domain. As can be seen from the table, the condition numbers remain bounded independently of the number of levels as predicted by the theory. In Table 7.2, we present the results from computations on the second domain. Even though Assumption (A.1) does not hold for this domain, we see that the condition numbers of the preconditioned system remain bounded. 
TABLE 7.1

Conditioning of $\mathbf{B}_{J} \mathbf{A}_{J}$ for the domain in Figure 7.1.

\begin{tabular}{|c|c|c|c|c|}
\hline $\begin{array}{c}\text { Level } \\
J\end{array}$ & $\begin{array}{c}\text { Minimum eigen- } \\
\text { value of } \mathbf{B}_{J} \mathbf{A}_{J}\end{array}$ & $\begin{array}{c}\text { Maximum eigen- } \\
\text { value of } \mathbf{B}_{J} \mathbf{A}_{J}\end{array}$ & $\begin{array}{c}\text { Condition } \\
\text { number }\end{array}$ & $\begin{array}{c}\text { Degrees of } \\
\text { freedom }\end{array}$ \\
\hline 2 & 0.59 & 1.13 & 1.92 & 67 \\
\hline 3 & 0.56 & 1.07 & 1.90 & 343 \\
\hline 4 & 0.52 & 1.09 & 2.10 & 1451 \\
\hline 5 & 0.47 & 1.10 & 2.34 & 5971 \\
\hline 6 & 0.45 & 1.10 & 2.48 & 24227 \\
\hline 7 & 0.44 & 1.10 & 2.52 & 97603 \\
\hline
\end{tabular}

TABLE 7.2

Conditioning of $\mathbf{B}_{J} \mathbf{A}_{J}$ for the domain in Figure 7.2.

\begin{tabular}{|c|c|c|c|c|}
\hline $\begin{array}{c}\text { Level } \\
J\end{array}$ & $\begin{array}{c}\text { Minimum eigen- } \\
\text { value of } \mathbf{B}_{J} \mathbf{A}_{J}\end{array}$ & $\begin{array}{c}\text { Maximum eigen- } \\
\text { value of } \mathbf{B}_{J} \mathbf{A}_{J}\end{array}$ & $\begin{array}{c}\text { Condition } \\
\text { number }\end{array}$ & $\begin{array}{c}\text { Degrees of } \\
\text { freedom }\end{array}$ \\
\hline 2 & 0.61 & 1.32 & 2.24 & 221 \\
\hline 3 & 0.54 & 1.16 & 2.14 & 911 \\
\hline 4 & 0.43 & 1.08 & 2.73 & 3731 \\
\hline 5 & 0.38 & 1.12 & 3.13 & 15131 \\
\hline 6 & 0.36 & 1.12 & 3.33 & 60971 \\
\hline
\end{tabular}

\section{REFERENCES}

[1] Y. Achdou And Y. A. Kuznetsov, Substructuring preconditioners for finite element methods on nonmatching grids, East-West J. Numer. Math., 3 (1995), pp. 1-28.

[2] F. Ben Belgacem, The mortar finite element method with Lagrange multipliers, Numer. Math., 84 (1999), pp. 2173-2197.

[3] C. Bernardi, Y. Maday, and A. T. Patera, Domain decomposition by the mortar element method, in Asymptotic and Numerical Methods for Partial Differential Equations with Critical Parameters, H. G. Kaper and M. Garbey, eds., Kluwer Academic Publishers, Dordrecht, The Netherlands, 1993, pp. 269-286.

[4] C. Bernardi, Y. Maday, and A. T. Patera, A new nonconforming approach to domain decomposition: The mortar element method, in Nonlinear Partial Differential Equations and Their Applications, H. Brezis and J. L. Lions, eds., Pitman Res. Notes Math. Ser. 299, Longman Scientific and Technical, Harlow, UK, 1994, pp. 13-51.

[5] D. Braess, W. Dahmen, and C. Wieners, A Multigrid Algorithm for the Mortar Finite Element Method, Technical Report 153, Institut für Geometrie und Praktische Mathematik, RWTH Aachen, Aachen, Germany, 1998.

[6] J. H. Bramble, Multigrid Methods, Pitman Res. Notes Math. Ser. 294, Longman Scientific and Technical, Harlow, UK, 1993.

[7] J. H. Bramble, J. E. PASciak, And J. XU, The analysis of multigrid algorithms with nonnested spaces or noninherited quadratic forms, Math. Comp., 56 (1991), pp. 1-34.

[8] J. H. Bramble and J. Xu, Some estimates for a weighted $L^{2}$ projection, Math. Comp., 56 (1991), pp. 463-476.

[9] J. H. Bramble and X. Zhang, The analysis of multigrid methods, in Handbook of Numerical Analysis, Vol. 7, 2000, to appear.

[10] S. Brenner and L. R. Scott, The Mathematical Theory of Finite Element Methods, Texts Appl. Math. 15, Springer-Verlag, New York, 1994.

[11] M. A. CASARIN AND O. B. WidLund, A hierarchical preconditioner for the mortar finite element method, Electron. Trans. Numer. Anal., 4 (1996), pp. 75-88.

[12] J. Descloux, On finite element matrices, SIAM J. Numer. Anal., 2 (1972), pp. 260-265.

[13] J. Gopalakrishnan, On the Mortar Finite Element Method, Ph.D. thesis, Texas A\&M University, College Station, TX, 1999.

[14] P. Grisvard, Elliptic Problems in Nonsmooth Domains, Monographs Stud. Math. 24, Pitman, Boston, London, 1985.

[15] R. B. Kellog, Interpolation Between Subspaces of a Hilbert Space, Technical Note BN-719, University of Maryland, College Park, MD, 1971. 
[16] J. L. Lions and E. Magenes, Non-Homogeneous Boundary Value Problems and Applications, Vol. I, Springer-Verlag, New York, Heidelberg, 1972.

[17] P. Oswald, Multilevel Finite Element Approximation, Teubner Skripten zur Numerik, B. G. Teubner, Stuttgart, 1994.

[18] Y. SAAD, Iterative Methods for Sparse Linear Systems, PWS, Boston, 1996.

[19] P. Seshaiyer AND M. SuRI, Uniform $h p$ convergence results for the mortar finite element method, Math. Comp., (2000), to appear.

[20] J. R. Shewchuk, Triangle: Engineering a $2 D$ quality mesh generator and Delaunay triangulator, in First Workshop on Applied Computational Geometry, Association for Computing Machinery, New York, 1996, pp. 124-133.

[21] V. Thomée, Galerkin Finite Element Methods for Parabolic Problems, Lecture Notes in Math. 1054, Springer-Verlag, Berlin, New York, 1984. 